\title{
Meningeal inflammation in multiple sclerosis induces phenotypic changes in cortical microglia that differentially associate with neurodegeneration
}

\author{
Lynn van Olst ${ }^{1}$. Carla Rodriguez-Mogeda ${ }^{1} \cdot$ Carmen Picon $^{2} \cdot$ Svenja Kiljan $^{3} \cdot$ Rachel E. James $^{2} \cdot$ Alwin Kamermans $^{1}$. \\ Susanne M. A. van der Pol ${ }^{1}$ - Lydian Knoop ${ }^{4}$. Iliana Michailidou ${ }^{5}$. Evelien Drost ${ }^{1}$. Marc Franssen ${ }^{1}$. Geert J. Schenk ${ }^{3}$. \\ Jeroen J. G. Geurts ${ }^{3}$. Sandra Amor ${ }^{4}$. Nicholas D. Mazarakis ${ }^{2} \cdot$ Jack van Horssen $^{1} \cdot$ Helga E. de Vries ${ }^{1,6}$. \\ Richard Reynolds ${ }^{2,7} \cdot$ Maarten E. Witte ${ }^{1,4}$
}

Received: 29 October 2020 / Revised: 15 February 2021 / Accepted: 3 March 2021 / Published online: 29 March 2021

(c) The Author(s) 2021

\begin{abstract}
Meningeal inflammation strongly associates with demyelination and neuronal loss in the underlying cortex of progressive MS patients, thereby contributing significantly to clinical disability. However, the pathological mechanisms of meningeal inflammation-induced cortical pathology are still largely elusive. By extensive analysis of cortical microglia in post-mortem progressive MS tissue, we identified cortical areas with two MS-specific microglial populations, termed MS1 and MS2 cortex. The microglial population in MS1 cortex was characterized by a higher density and increased expression of the activation markers HLA class II and CD68, whereas microglia in MS2 cortex showed increased morphological complexity and loss of P2Y12 and TMEM119 expression. Interestingly, both populations associated with inflammation of the overlying meninges and were time-dependently replicated in an in vivo rat model for progressive MS-like chronic meningeal inflammation. In this recently developed animal model, cortical microglia at 1-month post-induction of experimental meningeal inflammation resembled microglia in MS1 cortex, and microglia at 2 months post-induction acquired a MS2-like phenotype. Furthermore, we observed that MS1 microglia in both MS cortex and the animal model were found closely apposing neuronal cell bodies and to mediate pre-synaptic displacement and phagocytosis, which coincided with a relative sparing of neurons. In contrast, microglia in MS2 cortex were not involved in these synaptic alterations, but instead associated with substantial neuronal loss. Taken together, our results show that in response to meningeal inflammation, microglia acquire two distinct phenotypes that differentially associate with neurodegeneration in the progressive MS cortex. Furthermore, our in vivo data suggests that microglia initially protect neurons from meningeal inflammation-induced cell death by removing pre-synapses from the neuronal soma, but eventually lose these protective properties contributing to neuronal loss.
\end{abstract}

Keywords Multiple sclerosis $\cdot$ Microglia $\cdot$ Neurodegeneration $\cdot$ Meninges $\cdot$ Cortical pathology $\cdot$ Neuroinflammation

Lynn van Olst and Carla Rodriguez-Mogeda have contributed equally to this work.

Richard Reynolds

r.reynolds@imperial.ac.uk

Maarten E. Witte

m.e.witte@amsterdamumc.nl

Extended author information available on the last page of the article

\section{Introduction}

Multiple sclerosis (MS) is the most common chronic neurodegenerative and neuroinflammatory disease in young adults [8]. At disease onset, the majority of MS patients present with a relapsing-remitting disease course (RRMS), and despite availability of many disease-modifying therapies most RRMS patients will eventually develop secondary progressive MS (SPMS) [20, 28, 36]. The transition into SPMS is characterized by gradual worsening of neurological disability without periods of remission and currently no efficient therapeutic options exist for the majority of patients [16]. The main cause of disease progression in SPMS is 
neurodegeneration, which encompasses tissue damage in the white matter (WM) [29] and accumulating pathology in the grey matter (GM), including demyelination and loss of neurons and synapses in the cerebral cortex [25, 31, 41]. Multiple studies have shown that the degree of cortical pathology provides a better correlate for progression of clinical disability than the number of WM lesions $[5,6,15]$. The lack of therapies for SPMS can, therefore, be largely attributed to our incomplete understanding of the mechanisms behind cortical neurodegeneration. This, in turn, is severely hindered by absence of suitable animal models. However, a number of studies have now indicated that chronic, compartmentalized inflammation of the nearby leptomeninges is likely to drive many aspects of cortical pathology [23, 31].

Meningeal inflammation in SPMS is characterized by accumulation of immune cells, including B, T and myeloid cells, either diffusely present or in aggregates resembling tertiary lymphoid follicles [3, 48]. The degree of inflammation and the presence of follicles both associate with the severity of cortical pathology, possibly by production and subsequent diffusion of pro-inflammatory cytokines into the cortex $[32,46]$. Indeed, in a recently developed animal model for chronic meningeal inflammation, chronic overexpression of two of these cytokines, TNF $\alpha$ and IFN $\gamma$, in the leptomeninges of rats was recently shown to induce robust meningeal inflammation, cortical demyelination and neuronal loss as seen in SPMS [24].

Microglia are the brain-resident immune cells, and as such, they continuously scan their environment for structural damage or invading pathogens using their highly motile processes $[38,50]$. In addition, microglia are crucial for development and maintenance of neuronal networks by facilitating synaptic plasticity [55] and directly interacting with neuronal cell bodies to monitor and protect neuronal function [9]. Accordingly, upon brain insults, microglia actively adapt their shape and function to restore brain homeostasis [7, 10, 49, 50]. Despite this, multiple lines of evidence point towards an active contribution of microglia to neurodegeneration in many chronic neuroinflammatory and neurodegenerative diseases $[4,11]$. In both the previously mentioned in vivo model for chronic MS-like meningeal inflammation and in SPMS donors, meningeal inflammation strongly associates with activation of cortical microglia [24, 30]. However, it remains to be investigated whether cortical microglia augment the pro-inflammatory signal coming from the meninges and thereby contribute to neurodegeneration in SPMS cortex.

In the SPMS cortex, activated microglia have been found in close proximity to apical dendrites, neurites and neuronal soma, but whether these microglia contribute or try to salvage ongoing neurodegeneration is currently unknown [41]. To study the link between meningeal inflammation, microglial behavior and neurodegeneration in SPMS, we used post-mortem brain tissue from SPMS donors and a recently developed in vivo model for chronic MS-like meningeal inflammation [24]. We identified two subgroups of MS cortical areas with distinct patterns of microglial morphology, density, and protein expression. Interestingly, these two subgroups of MS cortex also showed separate levels of local meningeal inflammation and differed in the extent of neuronal damage. Highly similar microglial phenotypes and associations with neuronal damage were observed in vivo, where these phenotypes existed at different time points after the onset of progressive MS-like meningeal inflammation. Taken together, our data suggest that in progressive MS, chronic meningeal inflammation induces phenotypic changes in cortical microglia that differentially associate with cortical neurodegeneration in a time-dependent manner.

\section{Materials and methods}

\section{Human tissue samples}

Post-mortem fixed-frozen blocks containing cingulate or insular cortex gyrus from 20 confirmed secondary progressive MS (SPMS) cases (mean age of death $=48.9$, range 35-65) and six non-neuroinflammatory controls (mean age of death $=62$, range 35-77) were provided by the UK MS Society Tissue Bank at Imperial College London. All donors or their next of kin provided fully informed consent for autopsy and use of material for research under ethical approval by the National Research Ethics Committee (08/ MRE09/31). Relevant clinical and demographic information of individual control and SPMS cases are listed in Table 1. Tissue blocks were cut into 10 and $20 \mu \mathrm{m}$ sections, and stored at $-80{ }^{\circ} \mathrm{C}$ until further use. In some analyses, we had to omit 1 or 2 cases due to absence of meningeal tissue and upper cortical layers or loss of antigenicity in the corresponding tissue sections (listed in Table 1).

\section{Lentiviral vector production}

Lentiviral vectors carrying human TNF $\alpha$ and IFN $\gamma$ were produced as described previously [24]. In summary, HEK-293 cells were transfected with HIV-1 transfer plasmid (pRRLsincppt-CMV-TNF-WPRE or pRRL-sincppt-CMV-IFNWPRE genome plasmid), the packaging vector plasmids expressing HIV-1 gag/pol gene (pMD2-LgRRE), VSV-G envelope plasmids (pMD2-VSV-G) and HIV-1 Rev (pRSVRev) using $\mathrm{CaCl}_{2}$. Fresh medium supplemented with $10 \mathrm{mM}$ sodium butyrate was added after $16 \mathrm{~h}$. The supernatant was harvested and filtered through a $0.45 \mu \mathrm{m}$ filter after $36 \mathrm{~h}$, and centrifuged overnight. Lentiviral vectors were concentrated using ultracentrifugation and resuspended with TSSM (10 mM Tromethamine, $100 \mathrm{mM} \mathrm{NaCl}, 10 \mathrm{mg} / \mathrm{mL}$ sucrose 
Table 1 Clinical and demographic data

\begin{tabular}{|c|c|c|c|c|c|c|c|}
\hline Case ID & Gender $(\mathrm{m} / \mathrm{f})$ & $\begin{array}{l}\text { Age at } \\
\text { death } \\
\text { (year) }\end{array}$ & $\begin{array}{l}\text { Post-mortem } \\
\text { delay (hours) }\end{array}$ & Cause of death & $\begin{array}{l}\text { Disease } \\
\text { duration } \\
\text { (year) }\end{array}$ & $\begin{array}{l}\text { Time } \\
\text { progressive } \\
\text { (year) }\end{array}$ & $\begin{array}{l}\text { Time } \\
\text { wheelchair } \\
\text { (year) }\end{array}$ \\
\hline $\mathrm{C} 14$ & $\mathrm{~m}$ & 64 & 18 & Cardiac failure & NA & NA & NA \\
\hline $\mathrm{C} 25$ & $\mathrm{~m}$ & 35 & 22 & Carcinoma of the tongue & NA & NA & NA \\
\hline $\mathrm{C} 28$ & $\mathrm{f}$ & 60 & 13 & Ovarian cancer & NA & NA & NA \\
\hline C36 & $\mathrm{m}$ & 68 & 30 & Heart failure, fibrosing alveolitis, artherome & NA & NA & NA \\
\hline $\mathrm{C} 45$ & $\mathrm{~m}$ & 77 & 22 & Cardio pulmonary degeneration & NA & NA & NA \\
\hline $\mathrm{C} 48^{\mathrm{a}}$ & $\mathrm{m}$ & 68 & 10 & Metastatic colon cancer & NA & NA & NA \\
\hline MS330 & $\mathrm{f}$ & 59 & 21 & Pneumonia, MS & 39 & 24 & 21 \\
\hline MS342 & $\mathrm{f}$ & 35 & 9 & MS & 5 & 1 & 1 \\
\hline MS352 & $\mathrm{m}$ & 43 & 26 & Bronchopneumonia, MS & 18 & 14 & 10 \\
\hline MS371 & $\mathrm{m}$ & 40 & 27 & Pneumonia & 16 & 7 & 4 \\
\hline MS377 & $\mathrm{f}$ & 50 & 22 & Pneumonia, MS & 23 & 3 & Unknown \\
\hline MS402 & $\mathrm{m}$ & 46 & 12 & Pneumonia, MS & 26 & 9 & 7 \\
\hline MS403 & $\mathrm{f}$ & 54 & 11 & MS & 26 & 15 & 6 \\
\hline MS404 & $\mathrm{f}$ & 55 & 17 & Septicaemia, shock, pneumonia & 34 & 20 & 2 \\
\hline MS405 & $\mathrm{m}$ & 62 & 12 & Septicaemia, MS, metastatic colon cancer & 25 & 20 & 18 \\
\hline MS408 & $\mathrm{m}$ & 39 & 21 & Pneumonia, sepsis & 11 & 5 & 2 \\
\hline MS426 & $f$ & 48 & 21 & MS & 19 & 19 & 13 \\
\hline MS438 & $\mathrm{f}$ & 53 & 17 & MS & 12 & 4 & 4 \\
\hline MS445 & f & 62 & 13 & Lower respiratory tract infection, MS & 38 & 13 & 15 \\
\hline MS461 & $\mathrm{m}$ & 43 & 13 & Pneumonia, MS & 21 & 12 & 12 \\
\hline $\operatorname{MS} 473^{\mathrm{e}}$ & $\mathrm{f}$ & 39 & 9 & Pneumonia, MS & 13 & 10 & 6 \\
\hline MS513 & $\mathrm{m}$ & 51 & 17 & MS, respiratory failure & 18 & 15 & 14 \\
\hline $\operatorname{MS} 527^{f}$ & $\mathrm{~m}$ & 47 & 10 & Pneumonia, MS & 25 & 15 & 13 \\
\hline MS528 & $\mathrm{f}$ & 45 & 17 & MS & 25 & 10 & 3 \\
\hline MS530 & $\mathrm{m}$ & 42 & 15 & MS & 21 & 15 & 14 \\
\hline MS535 $^{\mathrm{h}}$ & $\mathrm{f}$ & 65 & 12 & MS & 40 & 25 & 20 \\
\hline
\end{tabular}

${ }^{a}$ Excluded from Figs. 2f, 3b,f and 5e

${ }^{\mathrm{b}}$ Excluded from Fig. 3e

${ }^{c}$ Excluded from Fig. 5e (all layers)

${ }^{\mathrm{d}}$ Excluded from Fig. $2 \mathrm{~g}$

${ }^{\mathrm{e}}$ Excluded from Fig. $2 \mathrm{f}$

${ }^{\mathrm{f}}$ Excluded from Fig. $2 \mathrm{~h}$

${ }^{\mathrm{g}}$ Excluded from Fig. 1c-f (layer 1)

${ }^{\mathrm{h}}$ Excluded from Fig. 5e (all layers)

and $10 \mathrm{mg} / \mathrm{mL}$ mannitol). The genome copy number was calculated using the Clontech Lenti-X qRT-PCR Titration kit (Takara Bio).

\section{Chronic meningeal inflammation animal model}

Eight- to ten-week-old female Dark Agouti rats (140-160 g) were obtained from Janvier Labs (France). Rats were housed in groups of four in a 12-h light/dark cycle and had ad libitum access to food and water. The UK Home Office approved all procedures. The induction of chronic meningeal inflammation (CMI) in Dark Agouti rats was performed as previously published [24]. Briefly, rats were anaesthetized with isofluorane and a subclinical autoimmune response to myelin was induced by injecting intra-dermally $5 \mu \mathrm{g}$ recombinant mouse myelin oligodendrocyte glycoprotein (MOG; amino acids 1-119) diluted in phosphate buffered saline (PBS) and emulsified in incomplete Freund's adjuvant (IFA, Sigma) or by injecting PBS alone emulsified in IFA as control (IFA ctrl). 20-23 days post-MOG or -IFA injection, rats underwent stereotactic surgery under isofluorane anesthesia. A 5-mm hole was drilled in the skull in the midline $0.9 \mathrm{~mm}$ caudal to bregma. A calibrated glass capillary needle attached to a fixed-needle $10 \mu \mathrm{L}$ Hamilton syringe 
was inserted to a depth of $2.3 \mathrm{~mm}$ down the sagittal sulcus, below the meningeal dura mater layer. The lentiviral vector mixture containing of $5 \times 10^{8}$ genomic copies of TNF $\alpha$ and $5 \times 10^{7}$ genomic copies of IFN $\gamma$ was injected with a rate of $0.20 \mu \mathrm{L} / \mathrm{mL}$. To allow diffusion of the mixture from the area of injection, the needle was left for $10 \mathrm{~min}$ in place and then slowly withdrawn. Under sodium pentobarbitone anesthesia, rats were perfused 28 and 56 days after lentiviral injection with PBS followed by $4 \%$ paraformaldehyde (PFA). Brains were subsequently removed, post-fixed in $4 \%$ PFA overnight, cryoprotected in $30 \%$ sucrose and cut into $10 \mu \mathrm{m}$ sections. Sections were stored at $-80^{\circ} \mathrm{C}$ until further use. Naïve animals that did not receive any injection and MOG-immunized rats without stereotactic viral injection were used as controls.

\section{Immunolabeling}

Sections were defrosted at room temperature and subjected to epitope retrieval at $95^{\circ} \mathrm{C}$ for $30 \mathrm{~min}$ followed by a blocking step at room temperature for $30 \mathrm{~min}$ with a solution containing $0.01 \%$ Triton X-100 (Sigma) and the appropriate $10 \%$ normal serum in PBS. Sections were incubated overnight at $4{ }^{\circ} \mathrm{C}$ with primary antibodies (for details, see Table 2) followed by a 2-h incubation in Alexa fluorophorelabeled secondary antibodies (Thermo Fisher Scientific) for immunofluorescence. All immunofluorescent-stained sections were counterstained for DNA using DAPI $(1: 10,000$,
Molecular Probes) followed by quenching of auto-fluorescence with $0.03 \%$ Sudan Black (Sigma) in 70\% ethanol for 5 min. After washing, sections were embedded in Mowiol mounting medium and stored in the dark at $4{ }^{\circ} \mathrm{C}$ until image acquisition. For immunohistochemistry, primary antibodies were visualized using the EnVision ${ }^{+}$visualization system with 3,3'-diaminobenzidine (DAB) as the chromogen (DAKO). Sections were subsequently counterstained with hematoxylin, dehydrated, and embedded in Entellan medium (Merck). Sections were stored at room temperature until image acquisition.

\section{Image acquisition and analysis}

All immunofluorescent-stained sections were imaged on a Nikon A1R laser-scanning confocal microscope equipped with a resonant scanner, except sections stained for synaptic markers which were imaged on a Leica TCS SP8 confocal microscope equipped with a white light laser and HyD hybrid detectors. In human tissue, all confocal images were taken in the cortex surrounding the deeper part of a sulcus, whereas in rats all images were taken in the sagittal sulcus and the cortex directly adjacent. Acquisition details for individual datasets are described in the corresponding sections below. DAB stained sections were scanned on a Vectra Polaris whole-slide scanner (Akoya Biosciences) using either a $20 \times(\mathrm{MOG})$ or $10 \times(\mathrm{HuC} / \mathrm{D})$ objective.

Table 2 Primary antibody details

\begin{tabular}{|c|c|c|c|c|c|c|}
\hline Antigen & Species & Used for & Dilution & Antigen retrieval & Manufacturer & Cat. Number \\
\hline CD19 & Rabbit & Human & $1: 100$ & EDTA-Tris pH 8 & Abcam & ab134114 \\
\hline CD3 & Mouse & Human & $1: 20$ & EDTA-Tris pH 8 & DAKO & M7254 \\
\hline CD3 & Mouse & Rat & $1: 500$ & without & BD Pharmigen & 550,295 \\
\hline CD4 & Rabbit & Human & $1: 150$ & Citrate $\mathrm{pH} 6$ & Abcam & ab133616 \\
\hline CD68 & Mouse & Human & $1: 100$ & Citrate $\mathrm{pH} 6$ & Abcam & Ab995 \\
\hline CD79a & Mouse & Rat & $1: 200$ & without & ThermoFisher & MA5-13,212 \\
\hline CD8 & Mouse & Human & $1: 1000$ & Citrate $\mathrm{pH} 6$ & DAKO & M7103 \\
\hline HLA class II (CR3/43) & Mouse & Human & $1: 500$ & Citrate $\mathrm{pH} 6$ & DAKO & M077501-2 \\
\hline HLA class II (OX6) & Mouse & Rat & $1: 50$ & without & In house & \\
\hline $\mathrm{HuC} / \mathrm{D}$ & Mouse & Human/Rat & $1: 1000$ & Citrate $\mathrm{pH}$ 6/EDTA-Tris $\mathrm{pH} 8$ & ThermoFisher & A21271 \\
\hline IBA1 & Goat & Human/Rat & $1: 500$ & Citrate $\mathrm{pH}$ 6/EDTA-Tris $\mathrm{pH}$ 8/without & Abcam & ab5076 \\
\hline IBA1 & Rabbit & Human/Rat & $1: 1000$ & Citrate $\mathrm{pH}$ 6/EDTA-Tris $\mathrm{pH}$ 8/without & WAKO & $019-19,741$ \\
\hline LAMP1 & Rabbit & Human & $1: 500$ & Citrate pH 6 & Cell Signaling & 9091P \\
\hline LAMP1 & Rabbit & Rat & $1: 1000$ & EDTA-Tris pH 8 & Abcam & $a b 24710$ \\
\hline MOG & Mouse & Human/Rat & $1: 50$ & Citrate $\mathrm{pH} 6$ & Reynolds Lab & N.A \\
\hline P2RY12 & Rabbit & Human & $1: 100$ & Citrate $\mathrm{pH} 6$ & AnaSpec & $55042 \mathrm{~A}$ \\
\hline P2RY12 & Rabbit & Rat & $1: 100$ & without & AnaSpec & $55043 \mathrm{~A}$ \\
\hline Synaptophysin & Mouse & Human/Rat & $1: 500$ & Citrate $\mathrm{pH}$ 6/EDTA-Tris $\mathrm{pH} 8$ & DAKO & M7315 \\
\hline TMEM119 & Rabbit & Human & $1: 500$ & Citrate $\mathrm{pH} 6$ & Atlas abcam & HPA051870 \\
\hline vGAT & Mouse & Rat & $1: 500$ & EDTA-Tris pH 8 & Synaptic Systems & 131011 \\
\hline
\end{tabular}




\section{Microglial morphology}

Confocal images were acquired from cortical layers 1,3 and $5 / 6$ in $20 \mu \mathrm{m}$ (human tissue) or $10 \mu \mathrm{m}$ (rat tissue) IBA1stained sections using a $40 \times$ objective and a $z$-stepsize of $1 \mu \mathrm{m}$ (human) or $63 \times$ objective and a z-stepsize of $0.1 \mu \mathrm{m}$ (rat). IBA $1^{+}$cells were manually traced from $2 \mathrm{D}$ maximum intensity projections of the aforementioned confocal images using FIJI (NIH). Per image, around 10-20 cells were selected for tracing. Cells were randomly selected, but had to meet several inclusion criteria: (1) cells should be completely included within the z-stack borders of the image; (2) cells should not overlap with one another; (3) cells should not be associated to a vessel. Next, traced microglia were analyzed using the Sholl Analysis Plugin [17] with a $0.3 \mu \mathrm{m}$ step size from the cell soma. Number of branches and junctions, and branch lengths were quantified by the AnalyzeSkeleton Plugin [1] using the same microglia cell tracings. Soma surface area of the traced microglia was measured using the freehand selection tool in FIJI.

\section{Microglial protein expression}

Confocal images were acquired in cortical layer 3 of sections double-labeled for IBA1 together with P2Y12, TMEM119, HLA class II or CD68 using a $40 \times$ objective and a z-stepsize of $0.5 \mu \mathrm{m}$. Per section, two images were taken. Imaris software (Version 9.5.2, Bitplane AG) was used to quantify mean fluorescence intensity in microglia, by first segmenting all IBA $1^{+}$microglia using the surfaces function followed by measuring the total fluorescence intensity sum of the aforementioned markers in microglia divided by total microglial volume. Final mean fluorescence intensity was determined as the average of both images.

\section{Meningeal immune cells}

3-6 confocal images were acquired in the meninges in and around sulci of sections stained for CD3/CD19, CD4/CD8 and IBA1 with a $20 \times$ objective and a z-step size of $1.4 \mu \mathrm{m}$. In rat sections stained for CD3/CD79a and IBA1, an image of the whole sagittal sulcus and its respective meninges was taken with a $20 \times$ objective and z-step size of $1 \mu \mathrm{m}$. The absolute number of positive cells and $\mathrm{DAPI}^{+}$nuclei within the meninges were counted manually using FIJI. Cells and nuclei located in meningeal vessels were excluded. To determine meningeal immune cell ratios, absolute $\mathrm{CD}^{+}$, $\mathrm{CD} 19^{+} / \mathrm{CD}_{79} \mathrm{a}^{+}$and $\mathrm{IBA} 1^{+}$cell numbers were normalized to the number of $\mathrm{DAPI}^{+}$nuclei in the same sections. Human sections containing 10 or fewer positive immune cells in meningeal tissue were excluded from the immune cell ratio quantifications.

\section{Demyelination and neuronal loss}

In whole-slide scans of MOG and $\mathrm{HuC} / \mathrm{D}$ stained sections, cortical layers were annotated using QuPath version v0.2.0$\mathrm{m} 7$ [2]. The annotated areas in MOG-stained sections were exported to FIJI, a manual threshold was set and the positive fraction per area was measured to determine $\mathrm{MOG}^{+}$ area in individual cortical layers. Secondly, in human MOGstained sections, all completely demyelinated cortical lesions were outlined in FIJI and used to calculate the percentage of demyelinated cortex. Neuronal density was measured using the positive cell detection function in QuPath. DAB threshold was manually adjusted for each image to account for differences in staining intensity and all other parameters were kept the same for each subject.

\section{Microglia-neuron association}

Confocal images were acquired from cortical layers 3 and $5 / 6$ in two different areas in sections co-stained for IBA 1 and $\mathrm{HuC} / \mathrm{D}$ with a $20 \times$ objective and z-stepsize of $1 \mu \mathrm{m}$. First, all $\mathrm{HuC} / \mathrm{D}^{+}$neuronal somata were marked in single-channel displays of each image using the Multi-Point tool in FIJI. The same tool was then used to mark all IBA $1^{+}$microglia cell bodies in the same images. The overlay image was used to count the amount of neuronal somata directly contacted by one or more microglial cell bodies, and vice versa. Only cell bodies containing a clearly visible $\mathrm{DAPI}^{+}$nucleus were counted.

\section{Peri-somatic synapse displacement}

Confocal images were taken from cortical layers 3 (human and rat) and 5/6 (human) of sections labeled for Synaptophysin, HuC/D and IBA1 using a $100 \times$ objective (human) or $63 \times$ objective and 2.0 digital zoom (rat) and a z-stepsize of $0.1 \mu \mathrm{m}$. In single-plane confocal images of $6 \mathrm{MS}$ brains and $3 \mathrm{CMI} 1$ and 2-month rats, $\mathrm{HuC} / \mathrm{D}^{+}$neuronal somata were outlined and their perimeter measured using FIJI. Next, neurons were classified as associated with $\mathrm{IBA} 1^{+}$microglia or not, and the length of the soma in contact with microglia was measured in FIJI. Lastly, all Synaptophysin ${ }^{+}$pre-synaptic contacts on the outlined neuronal soma were measured and used to calculate the percentage of the neuronal soma that was covered by pre-synapses.

\section{Pre-synapse phagocytosis and pre-synaptic density}

15-30 confocal images were acquired from cortical layer 3 of stained sections for DAPI/IBA1/LAMP1/Synaptophysin (human) and DAPI/IBA1/LAMP1/vGAT (rat) with a 100 $\times($ different $x>\times)$ (human) or $63 \times$ and 2.0 digital zoom (rat) and a z-stepsize of $0.1 \mu \mathrm{m}$. For pre-synaptic density 
and pre-synaptic engulfment by IBA $1^{+}$cells, 2 consecutive z-planes (human) or full z-stack images (rat) were used. Using Imaris software, surfaces marking nuclei $\left(\mathrm{DAPI}^{+}\right)$, microglia $\left(\mathrm{IBA} 1^{+}\right)$and lysosomes $\left(\mathrm{LAMP} 1^{+}\right)$were created with individual settings per donor to account for differences in staining intensity. Next, the spots function was used to detect pre-synapses (Synaptophysin ${ }^{+}$in human/vGAT ${ }^{+}$in rat) using a spot diameter of $0.5 \mu \mathrm{m}$ and including a quality filter which was individually set for each donor/animal. Pre-synaptic density was quantified by dividing the total number of detected spots by the total volume of the images. Microglial phagocytosis of pre-synapses was calculated by identifying spots that had a maximum distance of $0 \mu \mathrm{m}$ to $\mathrm{IBA}^{+}{ }^{+} \mathrm{LAMP}^{+}{ }^{+}$surfaces and dividing their number to the total volume of the images or the total number of spots, whereas phagocytosis of pre-synapse by non-microglial cells was quantified by counting spots with a maximum distance of 0 to IBA1 ${ }^{-}$LAMP1 $^{+}$surfaces. The same images were used to quantify microglial LAMP1 expression, which was done as described in the Microglial Protein Expression section above, although now the average of 15-30 images was used to calculate mean fluorescence intensity.

\section{Principal component analysis}

To explore if combining parameters of microglia morphology, phenotype and density could reveal different MS subgroups, we performed a principle component analysis (PCA) with scaling of the parameters using the web-based MetaboAnalyst (http://www.metaboanalyst.ca) [40]. We included measures for microglia morphology and shape (AUC derived from the sholl analysis and soma size), microglia protein expression (P2Y12, TMEM119, CD68, HLA class II) and microglial density. For this analysis, data from all analyzed layers was combined if applicable. The few missing values were estimated using the median value of the group. To detect and characterize subpopulations of MS cortical areas based on their microglia phenotype, $K$-means clustering $(k=3)$ was applied and differences in individual microglial parameters between the subgroups were corroborated with appropriate statistical testing.

\section{Statistics}

All analyzes were done blinded to disease or experimental condition. Graphpad Prism 8.2.1 was used for all statistical tests. First, we used Shapiro-Wilk and $F$ test to test for normality and equality of variances, respectively, and appropriate tests were selected accordingly. For comparing two experimental groups, unpaired two-tailed Student's $t$ test with or without Welch's correction for unequal variances, or Mann-Whitney test was used. For comparing more than two groups, we used two-tailed one-way analysis of variance
(ANOVA) with Tukey test for multiple comparisons, Welch ANOVA followed by Dunnett's T3 multiple comparisons test, or Kruskal-Wallis test followed by Dunn's multiple comparisons test. To examine the strength of association between two variables either Pearson correlation or Spearman correlation was used. Data were judged to be statistically significant when $p<0.05$ and, if significant, reported in the figures using the significance levels indicated in the figure legends.

\section{Results}

\section{Cortical microglia acquire a different morphological appearance in progressive MS}

To assess the involvement of microglia in cortical pathology in progressive MS patients, we first quantified microglial number and morphological complexity in cortical layers 1 , 3 and 5/6 using confocal microscopy on IBA $1^{+}$stained sections of MS brains and non-neurological controls (Fig. 1a, b). While the density of IBA $1^{+}$microglia in MS cortex was variable and not significantly different from controls (Fig. 1c), morphological analysis of a large number of individually traced microglia revealed a more ramified microglia morphology in MS cortex, as indicated by the area under the curve (AUC) of the Sholl analysis [17] (Fig. 1d, e). This morphological change was most evident in neuronal layer 3 (Fig. 1f) and was further corroborated on a subset of MS and control cases stained for TMEM119, a microglia-specific protein (Online Resource 1a-c). Both microglial density and morphology were positively associated throughout the different cortical layers (Online Resource 1d). Further analyzes of microglial morphology revealed an increase in the peak of the AUC, which displays the maximum number of intersections in the Sholl analysis, a larger reach of the branches (wingspan), and increased number and length of branches and number of junctions in MS microglia located in neuronal layer 3 (Fig. 1f and Online Resource 1e). Lastly, we observed a trend towards a larger microglial soma in all analyzed cortical layers of progressive MS brains (Fig. 1f). Absence of IBA $1^{+}$cells with an amoeboid morphology and co-expression of P2Y12 with virtually all non-vessel associated IBA $1^{+}$cells indicates limited infiltration of peripheral myeloid cells in MS cortex (Online Resource 1f).

\section{Heterogeneous microglial marker expression in the progressive MS cortex}

To further characterize cortical microglia, we quantified the expression of homeostatic microglia markers P2Y12 (Fig. 2a) and TMEM119 (Fig. 2b), and two commonly used activation markers, HLA class II (Fig. 2c) and CD68 (Fig. 2d), in IBA1 ${ }^{+}$microglia in cortical layer 3 . We found 

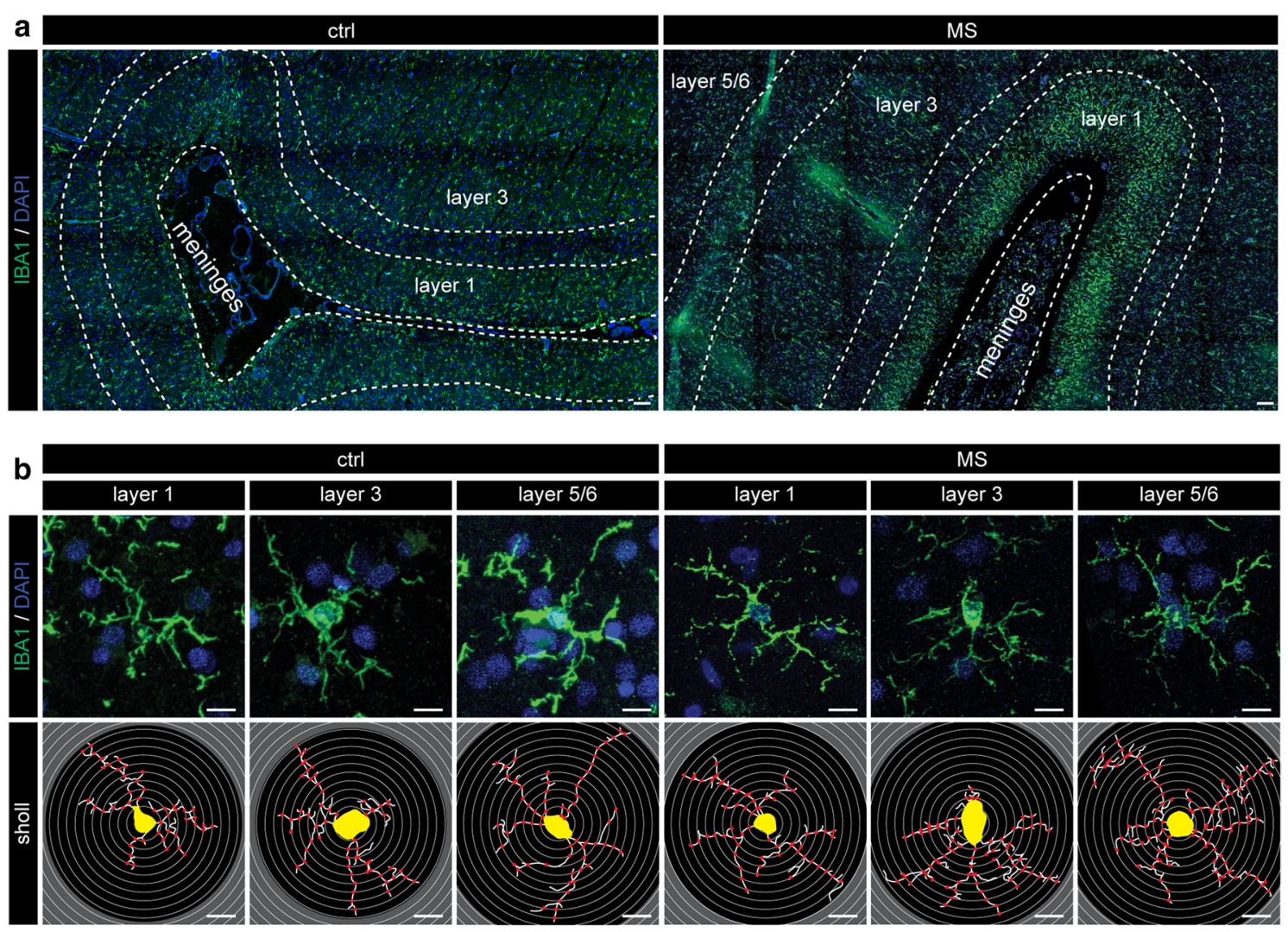

ctrl
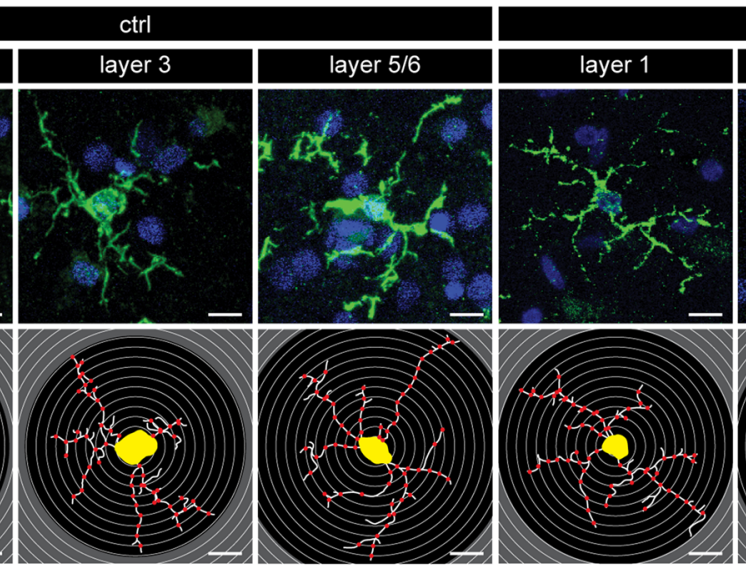

MS
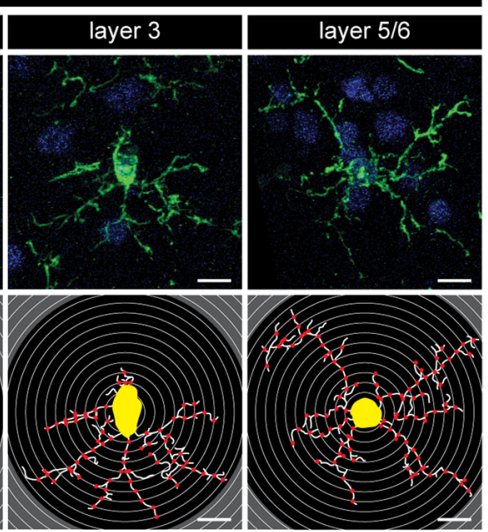

C

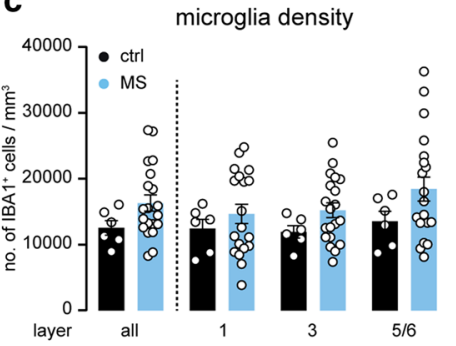

d

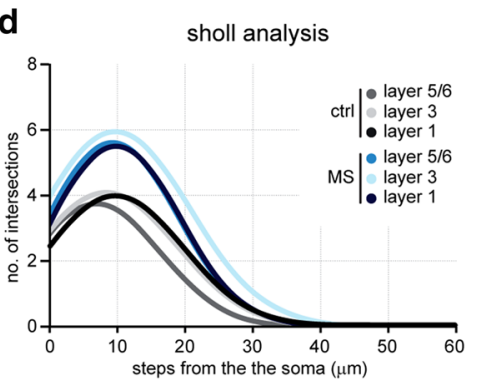

microglia morphology per donor

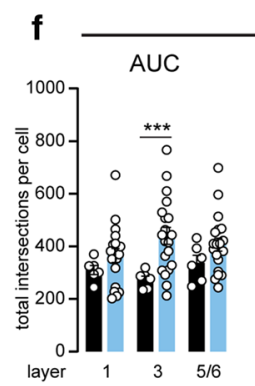

(a)

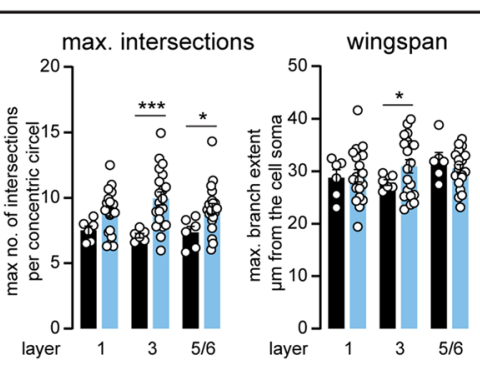

Fig. 1 Microglia morphology is altered in MS cortex. a Representative images displaying IBA1 expression in the cortex surrounding the sulcus of ctrl and MS subjects. b Micrographs of individual $\mathrm{IBA}^{+}{ }^{+}$microglia in cortical layer 1,3 and $5 / 6$ (top panels) and their corresponding traced outlines (bottom panels). c Quantification of the microglial density per cortical layer, quantified as the number of $\mathrm{IBA}^{+}$cells per $\mathrm{mm}^{3}$. d Non-linear curve fit of the average number of microglial branch intersections per $0.3 \mu \mathrm{m}$ step from the cell soma per cortical layer as measured by Sholl analysis. e Total Sholl-derived
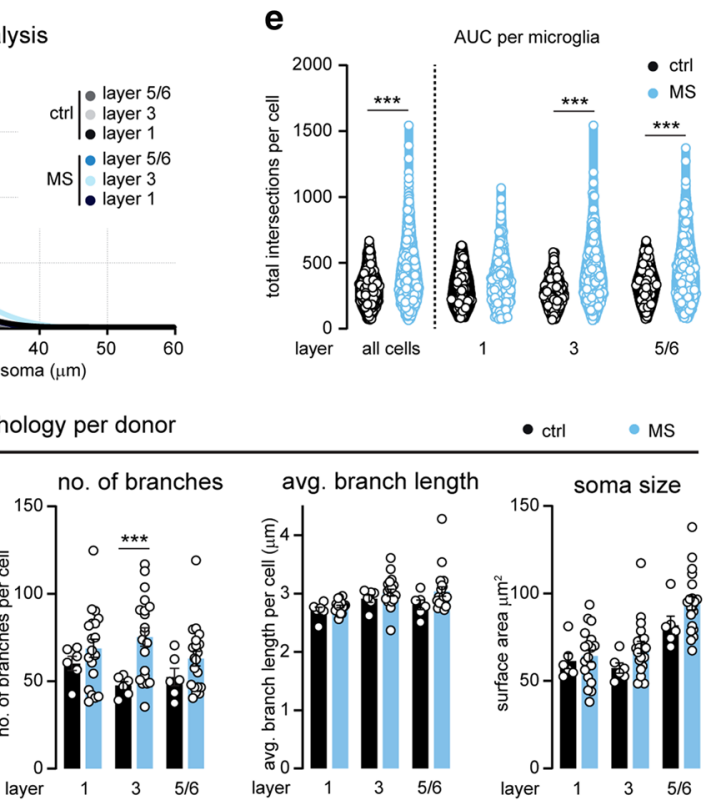

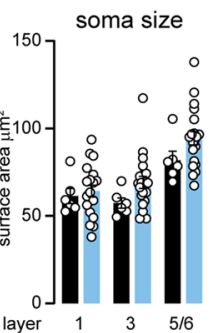

area under the curve (AUC) of individual microglia. f Quantification of different measurements (AUC, maximal number of intersections, wingspan, number of branches, average branch length, soma size) of microglial cell morphology averaged per donor. Individual datapoints indicate averaged data from an individual donor $(\mathbf{c}, \mathbf{f})$ or individual microglia (e), columns and error bars show mean \pm SEM; $* p<0.05$, $* * p<0.01$, *** $\mathrm{p}<0.001 ; n=6$ ctrls, $n=20$ MS subjects (c, d, f), $n=246$ microglia in ctrls, $n=1014$ microglia in MS (e); Scale bars $=10 \mu \mathrm{m}(\mathbf{a}), 100 \mu \mathrm{m}(\mathbf{b})$ 


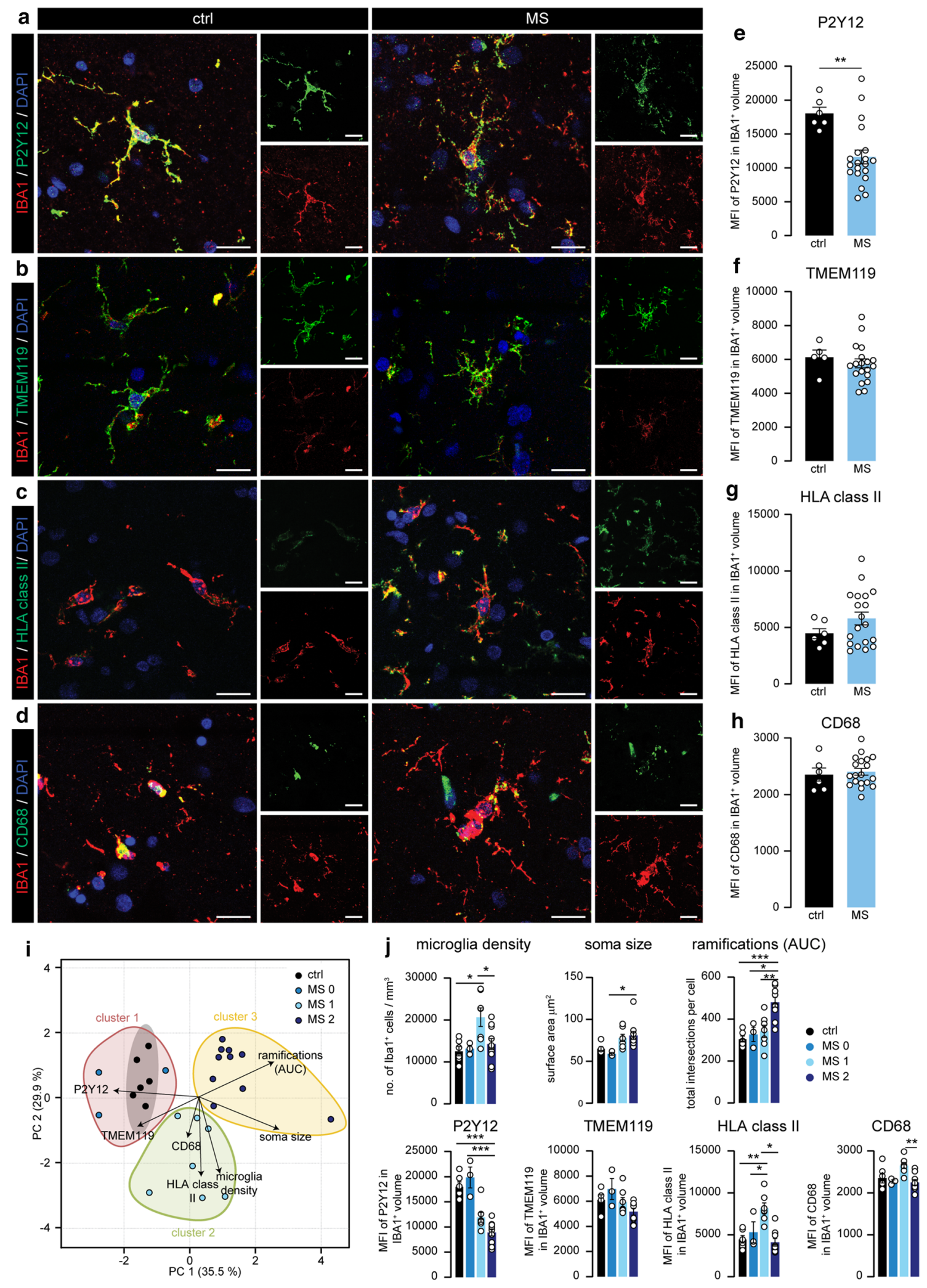


4Fig. 2 Differential expression of microglial markers in MS cortex corresponds with morphological changes. a-d Representative images displaying P2Y12 (a), TMEM119 (b), HLA class II (c) and CD68 (d) expression in IBA1 $1^{+}$microglia in cortical layer 3 of ctrl and MS donors. e-f. Quantification of the mean fluorescence intensity of P2Y12 (e), TMEM119 (f), HLA class II (g) and CD68 (h) in microglia. i. Scores for the first and second principle component (PC1/ PC2) of ctrl and MS cases. Subjects are coloured according to their K-means cluster assignment. j Quantification of microglia density, microglia soma size, Sholl-derived area under the curve, mean fluorescence intensity of P2Y12, TMEM119, HLA class II and CD68 in $\mathrm{IBA}^{+}$volume in the different subgroups. Individual datapoints indicate averaged data from an individual donor, columns and error bars show mean $\pm \mathrm{SEM} ;{ }^{*} p<0.05, * * p<0.01, * * * p<0.001 ; n=6 \mathrm{ctrls}$, except in (f) where $n=5$ ctrls, $n=20$ MS subjects; Scale bars $=20 \mu \mathrm{m}$

that P2Y12 expression was significantly lower in MS cortical microglia compared to controls (Fig. 2b), while we observed a trend towards increased microglial HLA class II expression (Fig. 2f). TMEM119 and CD68 were not differently expressed (Fig. 2h) although expression of all four markers was highly variable within the MS group.

\section{Cortical microglia morphology, density, and protein expression reveal two distinct MS subgroups}

To explore if the variation in both morphology and protein expression of cortical microglia within the analyzed MS cortical areas could be explained by the existence of subgroups with different microglial phenotypes, we used principle component analysis (PCA) and included parameters of microglia morphology, protein expression and cell density. PCA visualization showed the presence of three different subgroups separating MS and control cases based on their microglial phenotype (Fig. 2i). Then, using K-means clustering, MS cases were assigned to three distinct clusters which we named MS0, MS1, and MS2 (Fig. 2i). The three donors in the MS0 subgroup had a microglia phenotype that showed close resemblance to those in control (Fig. 2j). MS1 was mainly defined by low P2Y12, high HLA class II, high CD68 and a markedly increased microglia density, whereas MS2 was characterized by low P2Y12 expression and increased morphological complexity as quantified by Sholl analysis (AUC), higher number of junctions and branches and increased total branch length (Fig. $2 \mathrm{j}$ and Online Resource 2a). We did not find significant differences in age of death, disease duration, progressive MS duration and time in wheelchair between the MS subgroups (Online Resource $2 b)$. For the remainder of the study, we focused on MS-specific subgroups MS1 and MS2, as the MS0 group was limited $(n=3)$ and contained microglia that were similar to those in controls.
Increased meningeal T and B cells in progressive MS brains

Next, we explored whether meningeal inflammation is associated with the different progressive MS subgroups as several studies have already described meningeal inflammation as an important driver of cortical pathology in progressive MS $[23,31,38]$. We found that $\mathrm{CD} 19^{+} \mathrm{B}$ cells and $\mathrm{CD} 3^{+}$ $\mathrm{T}$ cells were increased in the meninges of progressive MS donors, while the levels of meningeal IBA $1^{+}$myeloid cells remained unchanged (Fig. 3a, b and Online Resource 3a). Interestingly, the increase in meningeal $\mathrm{CD} 19^{+} \mathrm{B}$ cells was most notable in the MS2 group (Fig. 3c), and correlated with increased morphological complexity of cortical microglia. To further dissect the involvement of meningeal $\mathrm{T}$ cells, we analyzed the presence of $\mathrm{CD} 4^{+}$and $\mathrm{CD} 8^{+} \mathrm{T}$ cells (Fig. 3d). As expected, both $\mathrm{CD} 4^{+}$and $\mathrm{CD} 8^{+} \mathrm{T}$-cell numbers were increased in a large subset of MS cases (Fig. 3e, and Online Resource 3b), and similar CD4/CD8 T-cell ratios shows that they were equally induced in the meninges overlying MS1 and MS2 cortex. (Fig. 3f).

\section{Experimental chronic meningeal inflammation time-dependently induces MS1- and MS2-like microglia}

To investigate whether meningeal inflammation could drive the microglial changes we observed in progressive MS cortex, we made use of a novel animal model of chronic experimental meningeal inflammation (CMI; Online Resource 4a) which has recently been shown to replicate important features of cortical pathology in progressive MS patients [24]. In this model, a subclinical autoimmune response to myelin was induced and lentiviral vectors carrying the TNF $\alpha$ and IFN $\gamma$ genes were injected in the sagittal sulcus below the meningeal dura mater layer. As expected, we observed an increase in meningeal $\mathrm{CD}^{+} \mathrm{T}$ cells, $\mathrm{IBA}^{+}$myeloid cells and $\mathrm{CD} 79 \mathrm{a}^{+} \mathrm{B}$ cells in the sagittal sulcus of CMI rats 1 and 2 months after lentiviral injection. Especially CD79a ${ }^{+}$ $\mathrm{B}$ cells were most strongly increased in numbers after 2 months (Fig. 4a-c and Online Resource 4b). Similar to the previous study, we did not observe significant differences in meningeal immune cell infiltration between CMI rats, which underwent subclinical MOG immunization prior to lentiviral injection; and IFA control rats, which were only injected with IFA prior to lentiviral injections (Online Resource 4c).

Next, we compared microglial density, morphology and protein expression in the cortex surrounding the sagittal sulcus in CMI rats with appropriate controls. Here, we found that after 1 month, CMI animals displayed an increased microglia density (Fig. 4d) and a less ramified morphology (Fig. 4e-i). In contrast, after 2 months, microglial density was almost back to control levels (Fig. 4b-d) whereas 

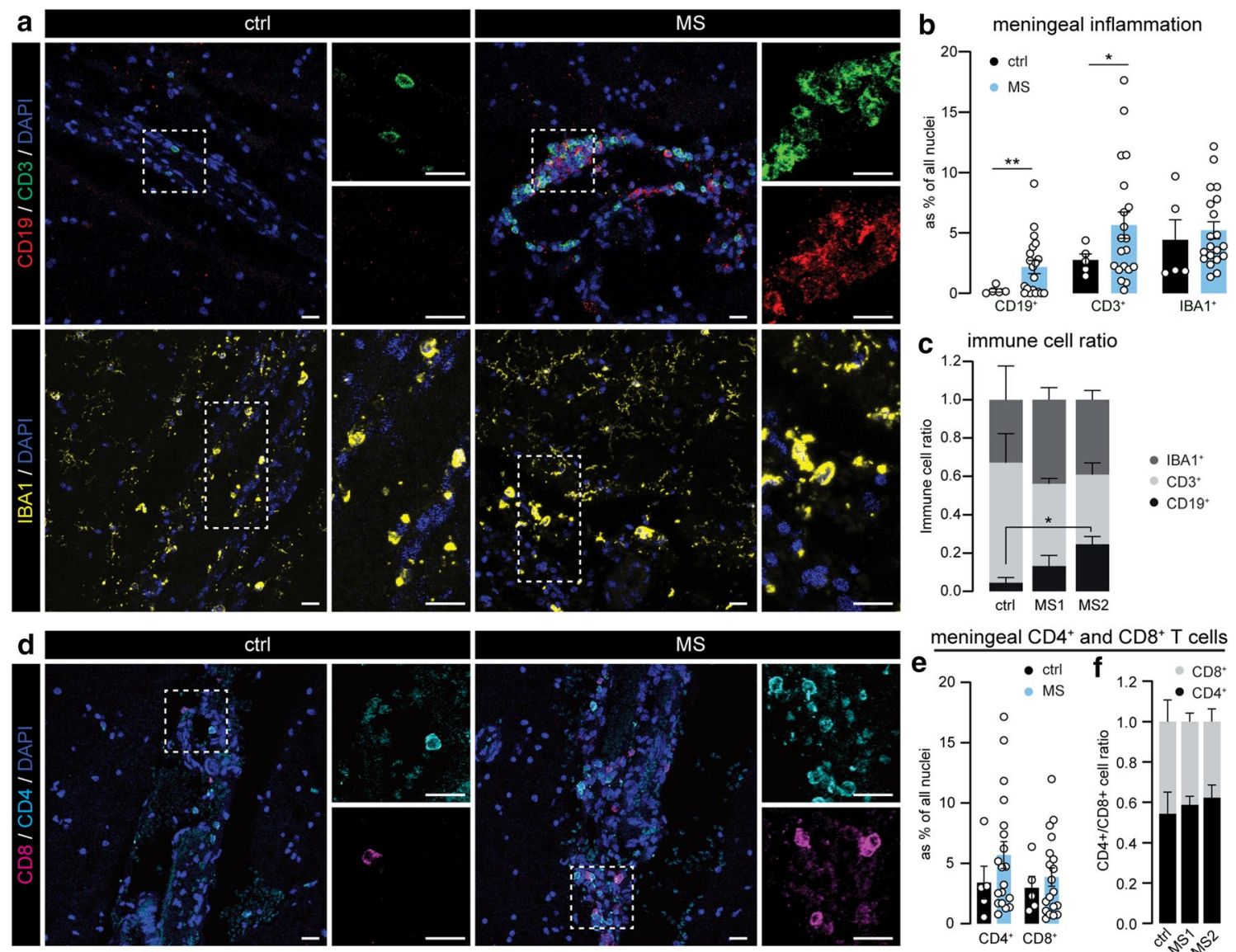

Fig. 3 MS2 microglia associate with meningeal B cells in progressive MS. a Representative images of the leptomeninges in ctrl and MS brains immunostained for CD3 (T cells), CD19 (B cells) and IBA1 (myeloid cells). b Quantification of $\mathrm{CD} 19^{+}, \mathrm{CD}^{+}$and $\mathrm{IBA}^{+}$cells as percentage of all nuclei in the meninges. c Immune cell ratio of $\mathrm{CD}_{1}{ }^{+}, \mathrm{CD}^{+}$and $\mathrm{IBA}^{+}$cells in the meninges of ctrls, MS1 and MS2 clusters. d Representative images of $\mathrm{CD}^{+}$(pink) and $\mathrm{CD} 4^{+}$ (cyan) $\mathrm{T}$ cells in meninges of ctrl and MS donors. e Quantification

morphological complexity of microglia was similar to or greater than in controls (Fig. 4e-f). We observed little to no changes in microglial soma size in both 1 and 2 month CMI animals (Fig S4d). Importantly, virtually all non-vesselassociated $\mathrm{IBA} 1^{+}$cells in the cortex of $\mathrm{CMI}$ rats were positive for P2Y12, indicating very little or absent infiltration of peripheral myeloid cells (Online Resource 4e). Quantification of P2Y12 and HLA class II expression in cortical layer 3 microglia of CMI rats (Fig. $4 \mathrm{j}-\mathrm{m}$ ) revealed a clear reduction in P2Y12 expression after both 1 and 2 months (Fig. 4j-i), whereas expression of HLA class II was highly upregulated in CMI 1 month microglia but almost back to levels seen in control animals after 2 months (Fig. $4 \mathrm{~m}$ ). We found similar changes in cortical microglial phenotype in IFA control animals after 1 and 2 months, though most changes were less pronounced than in the MOG-immunized CMI animals (Online Resource 4f). of $\mathrm{CD}^{+}$and $\mathrm{CD} 8^{+}$cells as percentage of all nuclei in the meninges. f $\mathrm{CD} 4^{+} / \mathrm{CD}^{+}$immune cell ratio in the meninges of ctrls, MS1 and MS2 clusters. Individual datapoints indicate averaged data from an individual donor, columns and error bars show mean \pm SEM; ${ }^{*} p<0.05,{ }^{*} p<0.01 ; n=5$ ctrls and $n=20$ MS subjects (b), $n=3$ ctrls, $n=5$ MS1 and $n=6$ MS2 (c), $n=5$ ctrls and $n=19$ MS subjects (e), $n=4$ ctrls, $n=7$ MS1 and $n=9$ MS2 (f); Scale bars $=20 \mu \mathrm{m}$

Taken together, cortical microglia in the CMI model at 1 month differ substantially from those at 2 months after lentiviral injection. Interestingly, microglia seen after 1 month carry many features of those seen in MS donors previously allocated to the MS1 subgroup, including a high microglial density, a less ramified morphology, low P2Y12 and high HLA class II expression. In addition, characteristics of CMI rats 2 months after injection, i.e. higher number of meningeal B cells, lower microglial density and HLA class II expression, but an increased morphological complexity, closely resembles the MS2 subgroup.

\section{MS1 and MS2 subgroups differentially associate with cortical neurodegeneration}

We next questioned whether the two MS clusters differentially associated with local tissue damage and for this 
purpose, we first quantified demyelination in MOG-stained sections. As expected, we observed that the majority of progressive MS tissues sections had one or more cortical lesions, mostly of the subpial type, which were completely absent from controls (Fig. 5a). However, we did not detect any differences between MS1 and MS2 cortical areas in either lesion load (as \% of total cortex; Fig. 5b) or $\mathrm{MOG}^{+}$area in the different cortical layers (Fig. 5c). We subsequently compared neuronal density per cortical layer using HuC/D immunolabeling (Fig. 5d). As it was recently reported that neurons in layer 2 and 3 of the cortex are especially prone to degenerate in progressive MS cortex [47], we also decided to include cortical layers 2 and 4 in this analysis. In line with a previous study [31], we observe a lower neuronal density in the upper cortical layers of the majority of progressive MS cases, but this only reached statistical significance in cortical layers 2 and 3 of MS2 cortex (Fig. 5e).

Several studies have described close contact between microglia and neurons in neuroinflammatory conditions $[13,49]$, leading to removal of pre-synaptic input from the neuronal soma. Here, using microglial IBA1 and neuronal HuC/D co-labeling, we show that in the MS1 cortex a higher percentage of neuronal somata was directly associated with one or more microglial cell bodies (Fig. $5 \mathrm{f}-\mathrm{g}$ ). Similarly, we also observed a higher percentage of microglia that were contacting neurons in both MS1 and MS2 (Online Resource 5a). Next, we found an almost complete loss of Synaptophysin $^{+}$(Syn) pre-synaptic input on the part of the neuronal soma that was occupied by a microglial cell body (Fig. 5h). In addition, we show an overall reduction of presynaptic input on neuronal soma in direct contact with one or more microglia in MS cortex (Fig. 5i).

Next, we assessed whether cortical microglia in MS1 and MS2 areas were engaged in phagocytosis of pre-synapses by quantifying the presence of $\mathrm{Syn}^{+}$structures in LAMP1 ${ }^{+}$ lysosomes in microglia (Fig. 5j). Similar to what happens in the thalamus of MS donors [54], we found a significant increase in pre-synaptic phagocytosis specifically in MS1 cortical microglia (Fig. 5j-k), which is in line with an overall increased phagocytic capacity of MS1 microglia as indicated by increased CD68 expression (Fig. 2) and corroborated here by increased LAMP1 expression (Online Resource $5 b)$. Remarkably, we did not observe a decrease in the total pre-synaptic density in layer 3 of either MS1 or MS2 cortex (Fig. 5l), which might be explained by the fact that the percentage of all pre-synapses that were located in microglial lysosomes was only around $0.3 \%$ (Online Resource $5 \mathrm{c}$ ). A small fraction of pre-synapses were located in lysosomes of non-microglial cells, but there was no difference between control, MS1 and MS2 cortex (Online Resource 5d).

\section{CMI induces time-dependent cortical neurodegeneration}

To assess associations with cortical tissue damage in CMI animals, we first measured demyelination in the cortical layers extending from the sagittal sulcus in MOG-stained sections (Fig. 6a). As shown previously, CMI induces cortical demyelination most strongly after 2 months and in the layers closest to the sulcus [24]. Due to the large variability of $\mathrm{MOG}^{+}$area in all groups, we did not detect any significant difference in layers 1 or 2 (Fig. 6b). Moreover, neuronal density in layer 1 was decreased at both time points, whereas we only found neuronal loss in layer 2 after 2 months (Fig. 6c, d).

In line with what we observed in MS1 cortical areas, the number of neuronal somata directly associated with microglia cell bodies was significantly increased in layer 3 of CMI 1 month rats, whereas after 2 months this was almost back to control levels (Fig. 6e-f). At both time points, these contacts resulted in removal of pre-synaptic input from the neuronal soma (Fig. 6g-h). Phagocytosis of vGAT ${ }^{+}$pre-synapses by microglia was increased after 1 and 2 months, albeit with a large variation between animals (Fig. 6i-j). Again, we did not observe a total loss of $\mathrm{vGAT}^{+}$pre-synapses in cortical layer 3 of CMI rats (Fig. 6k), possibly due to the small percentage of pre-synapses found in microglial lysosomes (Online Resource 6a). Furthermore, pre-synapse phagocytosis by non-microglia cells was not significantly different between groups (Online Resource 6b).

\section{Discussion}

Currently, therapeutic options for progressive MS patients are limited [16]. This is partly attributed to our lack of understanding of the pathological mechanisms driving the disease, which in turn is a consequence of a dearth of suitable animal models for progressive MS [33]. In this study, using both post-mortem tissue from progressive MS cases and a recently developed animal model for progressive MSrelated cortical pathology [24], we aimed to uncover mechanisms that drive neurodegeneration in progressive MS cortex and further establish the use of this new animal model to study underlying pathological processes.

Using extensive morphological analyses and quantification of several well-known markers for microglial activation, we could separate our cohort of progressive MS tissue into three distinct clusters. Cortices in two of these clusters (termed MS1 and MS2) contained a microglia population that significantly differed from controls. Microglia in MS1 cortical areas were characterized by a high cellular density and elevated expression of both HLA class II and CD68; whereas MS2 microglia were defined by a hyper-ramified 


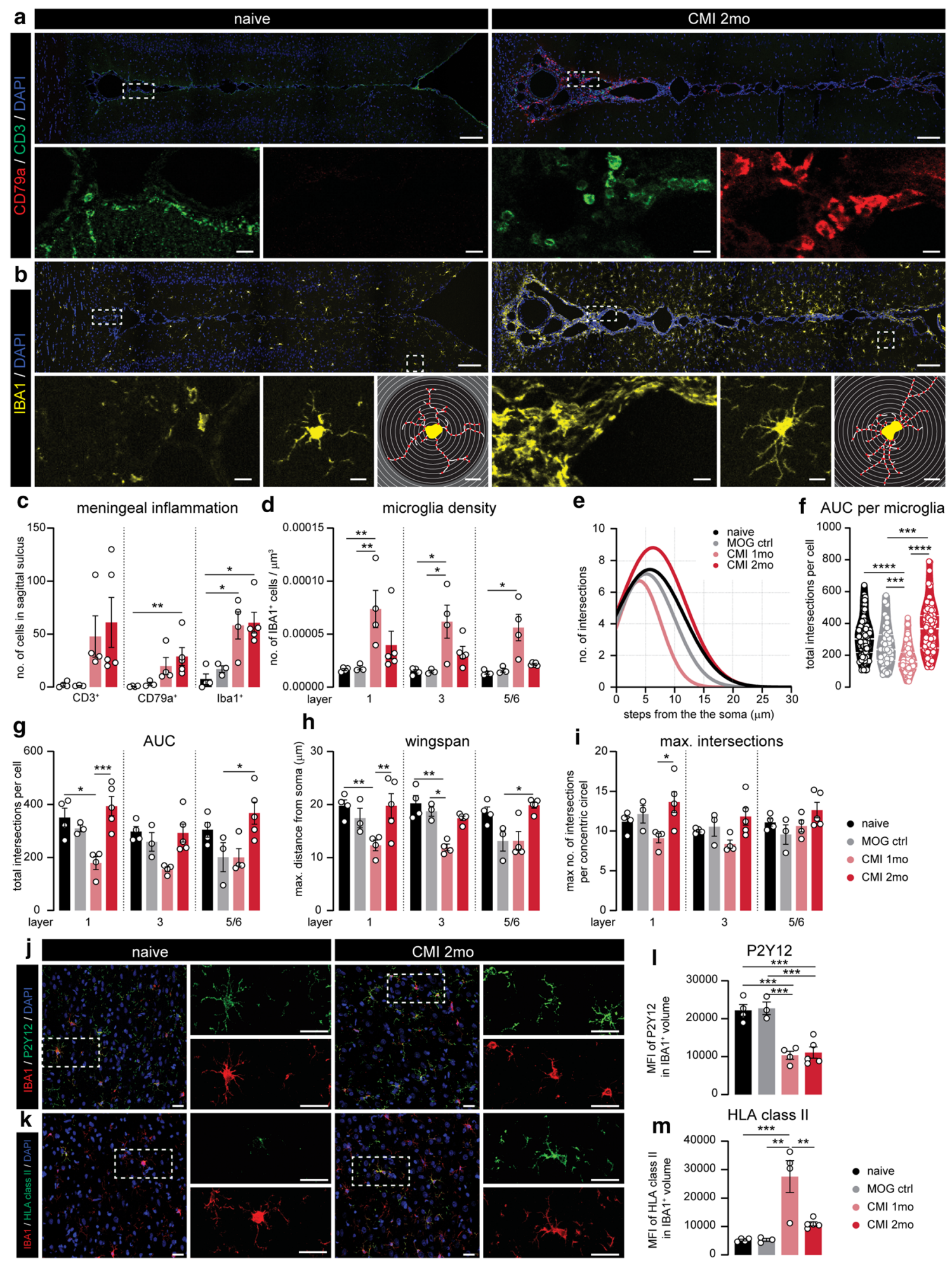

morphology and low P2Y12 expression. Both MS-specific microglial phenotypes were linked to increased meningeal inflammation, but only MS2 microglia associated with increased presence of $\mathrm{B}$ cells in the adjacent meninges. Remarkably, we detected cortical microglia that were very similar to both MS1 and MS2 in an animal model for chronic MS-like meningeal inflammation (CMI). Cortical microglia found 1 month after the induction of meningeal inflammation resembled microglia in MS1 areas, while microglia at 2 months after induction shared many features with MS2 
४Fig. 4 Experimental chronic meningeal inflammation induces similar microglial phenotypes as in MS cortex at different time points. a Representative image of CD3 (T cells) and CD79a (B cells) expression of the sagittal sulcus and surrounding cortex of naive and CMI 2 months animals. b Representative images of IBA1 expression in and around the sagittal sulcus of naïve and CMI 2 month rats (top panels). Higher magnification images of IBA1 expression inside the meninges (lower panel-left). Close-up of a single $\mathrm{IBA}^{+}$cell (lower panel-middle) and corresponding traced outline (lower panel-right). c Absolute number of $\mathrm{CD}^{+} \mathrm{T}$ cells, $\mathrm{CD} 79 \mathrm{a}^{+} \mathrm{B}$ cells and IBA $1^{+}$cells in the sagittal sulcus. d Microglial density per cortical layer, quantified as the number of $\mathrm{IBA}^{+}$cells per $\mathrm{mm}^{3}$ in the different animal groups e. Non-linear curve fit of the average number of microglial branch intersections per $0.3 \mu \mathrm{m}$ step from the cell soma per cortical layer as measured by the Sholl analysis. f. Total Sholl-derived area under the curve (AUC) of individual microglia. g-i Different measurements (AUC, wingspan, maximal number of intersections) of microglial cell morphology averaged per animal. j, k. Representative confocal images of cortical layer 3 from naïve and CMI 2 months displaying P2Y12 (j) or HLA class II (k) and IBA1 expression. i, m Mean fluorescence intensity of P2Y12 signal (i) and HLA class II (m) in IBA $1^{+}$ volume. Individual datapoints indicate averaged data from an individual donor $(\mathbf{c}-\mathbf{e}, \mathbf{g}-\mathbf{i}, \mathbf{l}, \mathbf{m})$ or individual microglia $(\mathbf{e})$, columns and error bars show mean \pm SEM; $* p<0.05, * * p<0.01, * * * p<0.001$; $n=4$ naïve, $n=3$ MOG ctrl, $n=4$ CMI $1 \mathrm{mo}, n=5$ CMI 2 months (ce, $\mathbf{g}-\mathbf{i}, \mathbf{l}, \mathbf{m}), n=112$ naïve, $n=43$ MOG ctrl, $n=173$ CMI 1 month, $n=143$ CMI 2 months (f); Scale bars $=100 \mu \mathrm{m}$ (top panels of $\mathbf{a}, \mathbf{b}$ ), $10 \mu \mathrm{m}$ (lower panels of $\mathbf{a}, \mathbf{b}), 25 \mu \mathrm{m}(\mathbf{j}, \mathbf{k})$

microglia. We further show that MS1 microglia spatially associate with neuronal cell bodies, whereas significant neuronal loss was restricted to the MS2 cortex. The spatial association between microglia and neurons was related to the removal of pre-synapses from the neuronal soma, and accompanied by increased pre-synapse phagocytosis by MS1 microglia. Again, neuronal changes in CMI animals reflected what we observed in progressive MS tissue, with cortical microglia 1 month after induction of meningeal inflammation closely apposing neuronal somata, and neuronal loss in the upper cortical layers being most prominent after 2 months. Furthermore, we observed a removal of presynapses from the soma of neurons that were in close contact with microglia in CMI animals and found evidence for pre-synapse phagocytosis by microglia at both time points.

Given the striking similarities between the two MSspecific clusters and the cortex of CMI animals after 1 and 2 months, it is tempting to speculate that the changes observed in MS1 cortex are caused by early stage meningeal inflammation and those observed in MS2 cortex by more chronic meningeal inflammation. As B cells are most prominent in the meninges of both MS2 cortex and 2-month CMI animals, this would suggest that at first $\mathrm{T}$ cells and myeloid cells populate the MS meninges, with the number of meningeal B cells rising over time. This also fits with the finding that the number of meningeal $\mathrm{B}$ cells and levels of B-cell-related cytokines in the cerebrospinal fluid associate with disease progression [30, 32, 45]. Furthermore, the extent of meningeal inflammation in MS donors, and more specifically the number of meningeal B cells, has been strongly linked to the presence of tertiary lymphoid-like follicles $[3,30]$. Unfortunately, we could identify too few of these lymphocyte clusters in the meninges of our tissue cohort to test whether they indeed are found predominantly in the MS2 cluster, as one would expect. Likewise, it would be interesting to explore whether these follicles also occur in CMI animals, which could open up studies examining their development and, eventually, used to find therapeutics that could block their formation.

Similar to findings under different neuroinflammatory conditions [7, 13], we show that the juxtaposition of microglia to neuronal somata leads to displacement of pre-synapses from the cell soma. At the same time, we observed increased pre-synapse phagocytosis by microglia. Interestingly, both displacement and phagocytosis of pre-synapses was predominantly seen in MS1 cortex. In contrast, significant neuronal loss was restricted to cortical layers 2 and 3 of MS2 cases, which is in line with a recent study showing a specific loss of $\mathrm{CUX2}^{+}$neurons in the same layers, which spatially associated with inflamed meninges [47]. Given the differences in neuronal pathology between MS1 and MS2 areas, we speculate that pre-synaptic displacement and stripping by MS1 microglia could represent a protective response to neuroinflammation and prevent substantial neuronal loss, as has previously been observed during LPS-induced neuroinflammation [7]. In contrast, MS2 microglia might have lost their ability to protect neurons from degenerating and perhaps even actively contribute to neuronal damage. Alternatively, it might be that pre-synaptic removal and the more pro-inflammatory profile (decreased P2Y12 and increased HLA class II and CD68 expression) of microglia in MS1 cortex is damaging to neurons but to such an extent that this not yet leads to an observable drop in neuronal density. The latter explanation seems to be supported by our recent finding of necroptotic neuronal death in non-immunized 1-month CMI rats. [43]. However, the number of neurons undergoing necroptosis at 1 month after injection is very small $\left(<5\right.$ per $\left.\mathrm{mm}^{2}\right)$, which might be due to the presence of neuroprotective MS1-like microglia. Hence, future research is needed to investigate whether these dichotomous microglial phenotypes are neuroprotective/neurodegenerative and whether they are mainly driven by differences in the neuroinflammatory environment, e.g. more meningeal B cells in MS2 cases, or the result of microglial exhaustion over time.

Surprisingly, we did not detect an overall decrease in pre-synaptic density in SPMS cortex, despite evidence for increased pre-synapse phagocytosis by microglia. Although loss of synapses has been extensively reported in hippocampus [14, 35], thalamus [54] and spinal cord of MS donors [42], evidence for a loss of synapses in the cortex is more ambiguous. Reduced spine density on apical dendrites, indicating post-synaptic loss, has been shown in both myelinated 


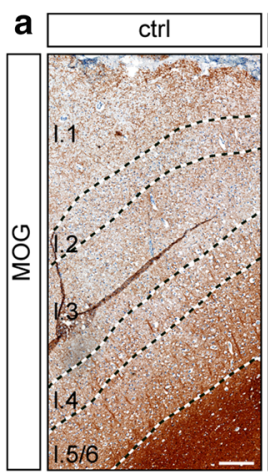

b demyelination
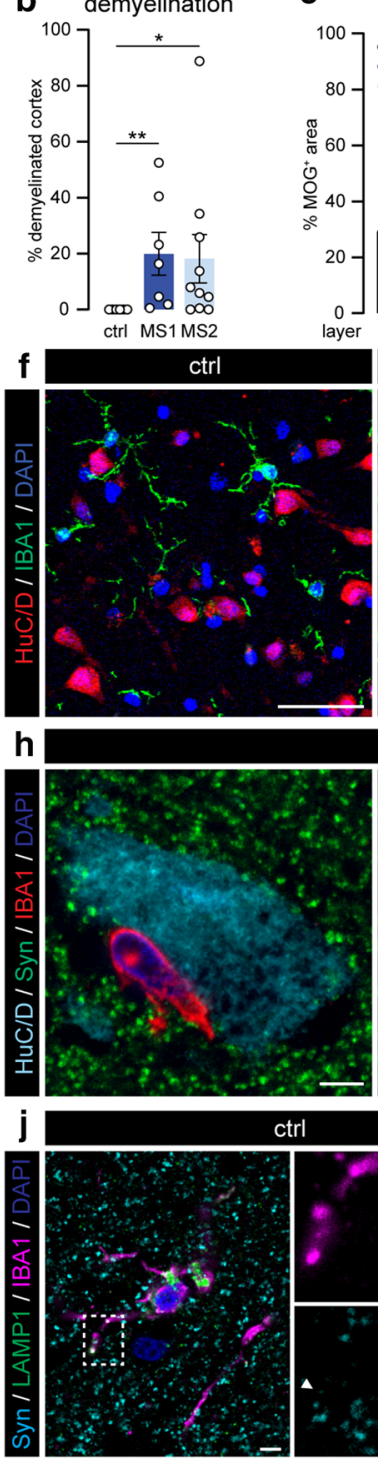

C

trl
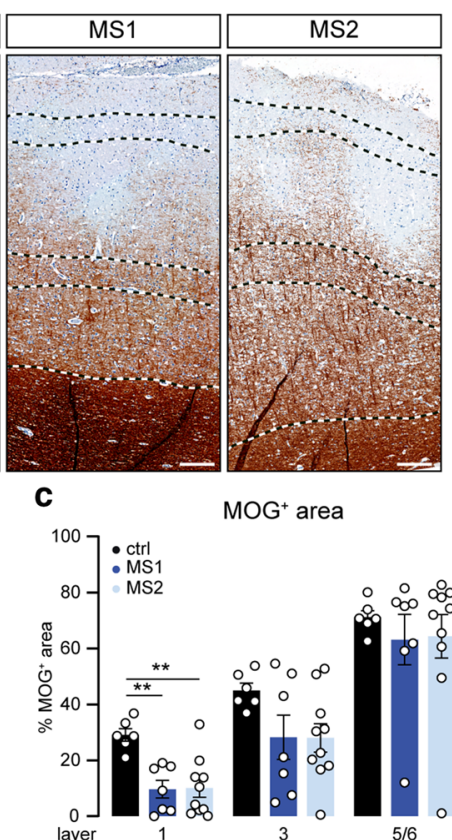

MS1

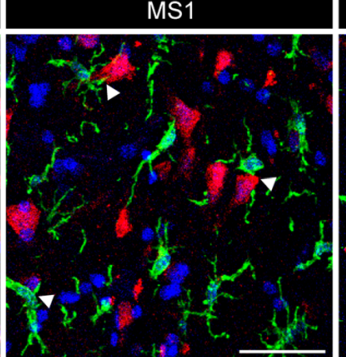

MS1
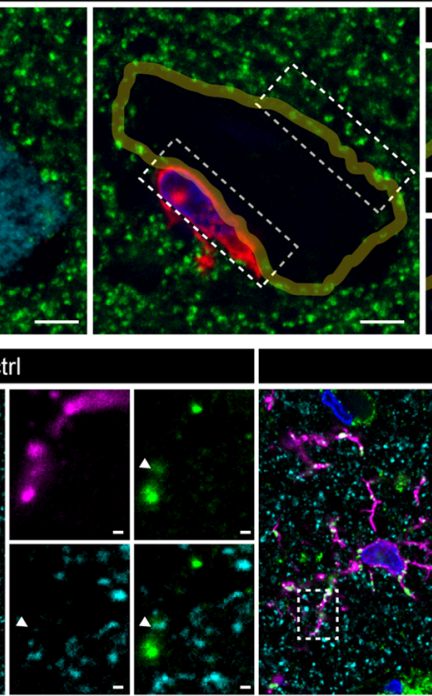

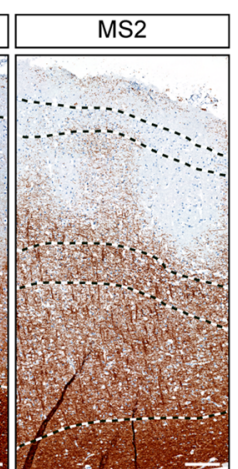

$\mathrm{MOG}^{+}$area
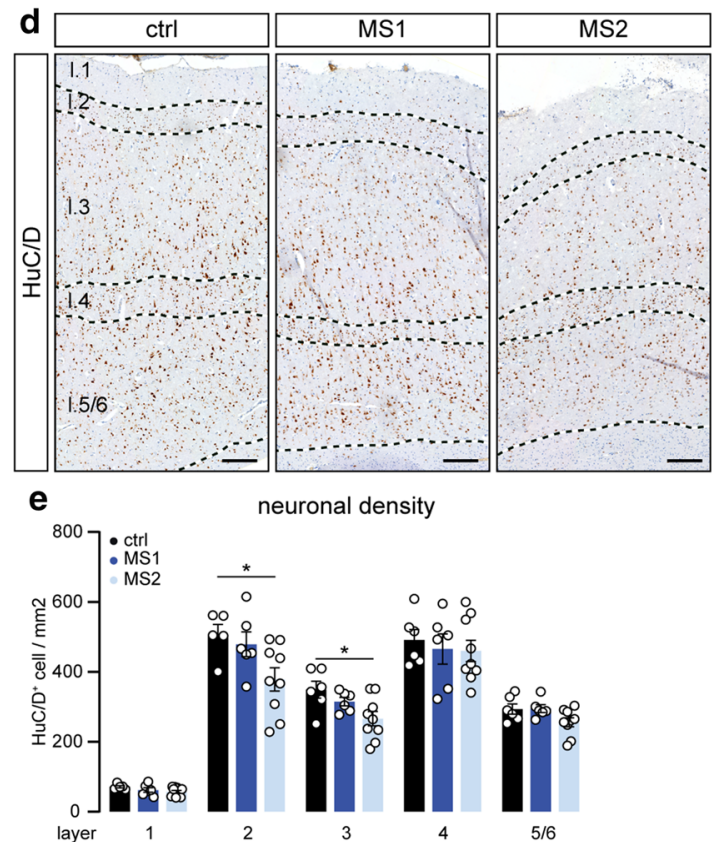

layer

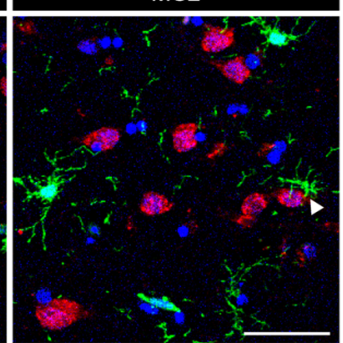

non-contacted soma

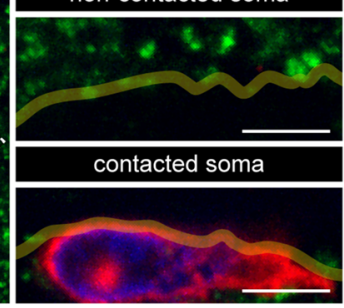

MS

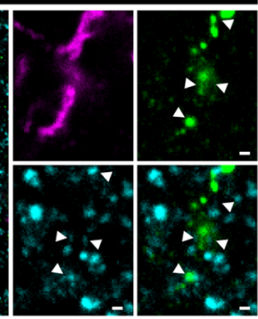

9 microglia-associated neurons
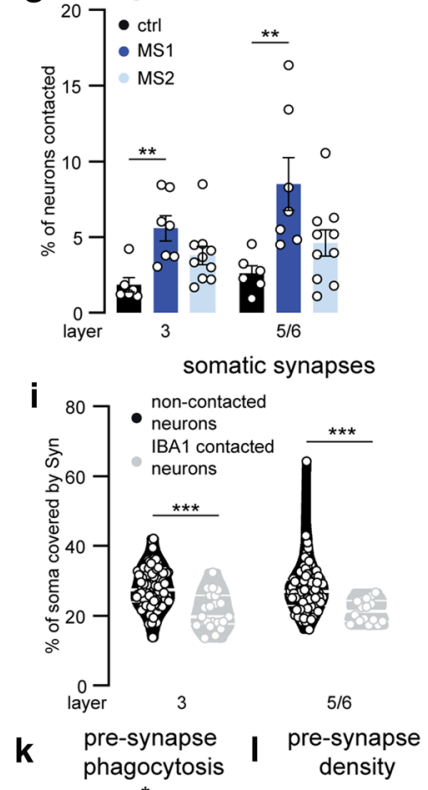

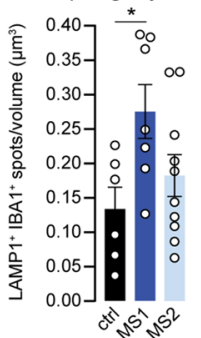

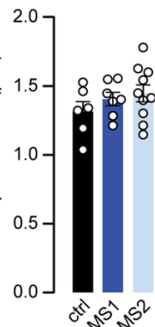

and demyelinated cortex of MS donors [25]. Whereas other studies, using similar methods to ours, only found a significant loss of Synaptophysin in leukocortical demyelinated lesions but not in normal appearing MS cortex [53] and no correlation with cortical atrophy [44]. Furthermore, the lack of decreased pre-synaptic density in our cohort might be explained by the low rate of pre-synapse removal $(<0.5 \%$ of pre-synapses within microglial lysosomes) in progressive MS cortex, which might allow for compensatory synaptogenesis. Alternatively, concomitant cortical thinning in 
4Fig. 5 MS subgroups based on microglia phenotype reveal different levels of neuronal loss and pre-synaptic removal. a Representative images of MOG-stained cortex of ctrl, MS1 and MS2 subjects. b, c Percentage of total cortical demyelination (b) and $\mathrm{MOG}^{+}$pixels (c) in cortical layers 1, 3 and 5/6 d. Representative images showing HuC/Dlabelled neurons in the cortex of ctrl, MS1 and MS2 subjects. e Quantification of neuronal density in the different cortical layers. $\mathbf{f}$ Representative images of cortical layers 5/6 from a ctrl, MS1 and MS2 case double-labelled with IBA1 (microglia) and HuC/D (neurons). Arrow heads depict soma-soma contact between microglia and neurons. $\mathrm{g}$ Percentage of neuronal somata directly contacted by microglia in cortical layers 3 and 5/6. h Representative single z-plane of a cortical layer 3 neuron from an MS1 subject immunolabelled for HuC/D (neuronal soma), IBA1 (microglia) and Synaptophysin (pre-synapse) (left panel). Yellow line depicts the outline of the neuronal cell body (middle panel), highlighting the synaptic input on the soma. Right panels show close-ups of the areas outlined in the middle panel. i Quantification of the percentage of neuronal soma covered by Synaptophysin ${ }^{+}$ structures in microglia-contacted neurons and neurons not associated with microglia in the MS cortex (pooled data from $3 \mathrm{MS} 1$, and 3 MS2 cases). j Representative images of cortical layer 3 from a ctrl and MS1 subject-stained for IBA1 (microglia), LAMP1 (lysosomes) and Synaptophysin (pre-synapses). $\mathbf{k}$ Number of Synaptophysin ${ }^{+}$ spots in microglial lysosomes per volume of cortical layer 3. 1 Total Synaptophysin $^{+}$spot density in cortical layer 3 . Individual datapoints indicate averaged data from an individual donor $(\mathbf{b}, \mathbf{c}, \mathbf{e}, \mathbf{g}, \mathbf{k}, \mathbf{l})$, or individual neurons (i), columns and error bars show mean $\pm \mathrm{SEM}$; ${ }^{*} p<0.05, * * p<0.01, * * * p<0.001 ; n=6$ ctrls $(\mathbf{b}, \mathbf{c}, \mathbf{e}, \mathbf{g}, \mathbf{k}, \mathbf{l})$ except in layer 1 (c, e) and layer 2 (e) where $n=5 \mathrm{ctrls}, n=7$ MS1 and $n=10$ MS2 (b, c, g, k, l), $n=6 \mathrm{MS} 1$ and $n=9 \mathrm{MS} 2(\mathbf{e}), n=110$ layer 3 and $n=102$ layer 5/6 neurons (i); Scale bars $=250 \mu \mathrm{m}(\mathbf{a}, \mathbf{d}), 50 \mu \mathrm{m}(\mathbf{f})$, $10 \mu \mathrm{m}(\mathbf{h}, \mathbf{j})$

these patients might obscure a reduction in synapse density despite an overall loss of synapses. Similarly, we also did not observe a significant difference in parameters of disease progression between the MS clusters, which one might expect given the differences in meningeal inflammation and neuronal loss and their strong correlation with disease severity [23, 31]. However, this could be a limitation of our experimental setup, in which we only analyzed one cortical region in each patient. And given the strong spatial association between meningeal inflammation, microglial activation and neuronal damage [31,47], pathology in this cortical region might not be predictive for the extent of tissue damage in the remaining cortex.

Microglia have been extensively studied in the context of age-related neurodegenerative diseases and white matter lesions in MS (for review see [4, 11]), however, their role in MS-related cortical pathology remains relatively obscure. Studies in amyotrophic lateral sclerosis (ALS), Alzheimer's disease (AD) and white matter MS lesions and their respective animal models have identified what appears to be a shared microglial response to (neuronal) damage, which involves retraction of their processes and significant changes in protein and/or mRNA expression. This phenotype has been termed either MGnD (microglia neurodegenerative phenotype) [27] or DAM (disease-associated microglia) [26]. Whether these microglial phenotypes are beneficial or detrimental remains a matter of debate and is likely time and disease-dependent. Here, we show that cortical microglia in MS respond rather differently than in ALS and $\mathrm{AD}$ and acquire an almost hyper-ramified morphology throughout the cortex, especially in the MS2 cortex. Similarly, we found that microglial activation markers, HLA and CD68 [34, 52], are not altered in MS2 microglia. Lastly, the expression of the homeostatic marker TMEM119 did not significantly differ from controls in all clusters unlike in MGnD/DAM microglia, which lose TMEM119 expression [26, 27]. Interestingly, hyper-ramified microglia have previously been observed in animal models of ischemic stroke [37] and chronic stress [21, 22, 51], which might be mediated by specific neuron-microglia interactions. For instance, increasing neuronal activity with neurotransmitter agonists can induce hyper-ramification of microglia via ATP signaling [12, 18, 39], while depleting CX3CR1 in microglia, which binds to neuronal fractalkine, yielded mice resistant to stress-induced microglial hyper-ramification [21]. As we could replicate the microglial alterations seen in both MS1 and MS2 cortex by experimentally inducing chronic meningeal inflammation in vivo, we propose that these alterations are caused by MS-related meningeal inflammation directly, through cytokine signaling for instance, and indirectly, via altered neuronal function. Since CMI is induced by chronic overexpression of both TNF $\alpha$ and IFN $\gamma$ in meninges of the sagittal sulcus, and TNF $\alpha$ and IFN $\gamma$ are both extensively produced in inflamed MS meninges [19], we assume that these cytokines are involved in driving the phenotypic changes in microglia. Future research should elucidate whether the difference between MS1 and MS2 microglia is caused by (1) a difference in duration of exposure to TNF $\alpha$ and IFN $\gamma,(2)$ involvement of other (B-cell derived) inflammatory factors, and/or (3) altered neuron-microglia signaling.

In this study, the results obtained from what we coined the CMI animal model, corroborate the initial experiments using this animal model [24], including persistent meningeal inflammation, cortical demyelination and neuronal loss. Although our data indicate that MOG immunization prior to lentiviral injection does not significantly alter the extent of meningeal inflammation, we did find that the difference between 1- and 2-month microglia was exacerbated in MOG-immunized rats, which is why we decided to focus on these animals. It would be interesting to explore if this difference is caused by increased cortical demyelination in MOG-immunized animals [24], or by the presence of MOGprimed lymphocytes in the meninges.

Taken together, we convincingly show that the experimental model of chronic meningeal inflammation closely mimics meningeal inflammation-induced cortical pathology in progressive MS patients. Although this model strongly emphasizes the role of TNF $\alpha$ and IFN $\gamma$ in driving cortical pathology, and thereby likely over-simplifies the complex 

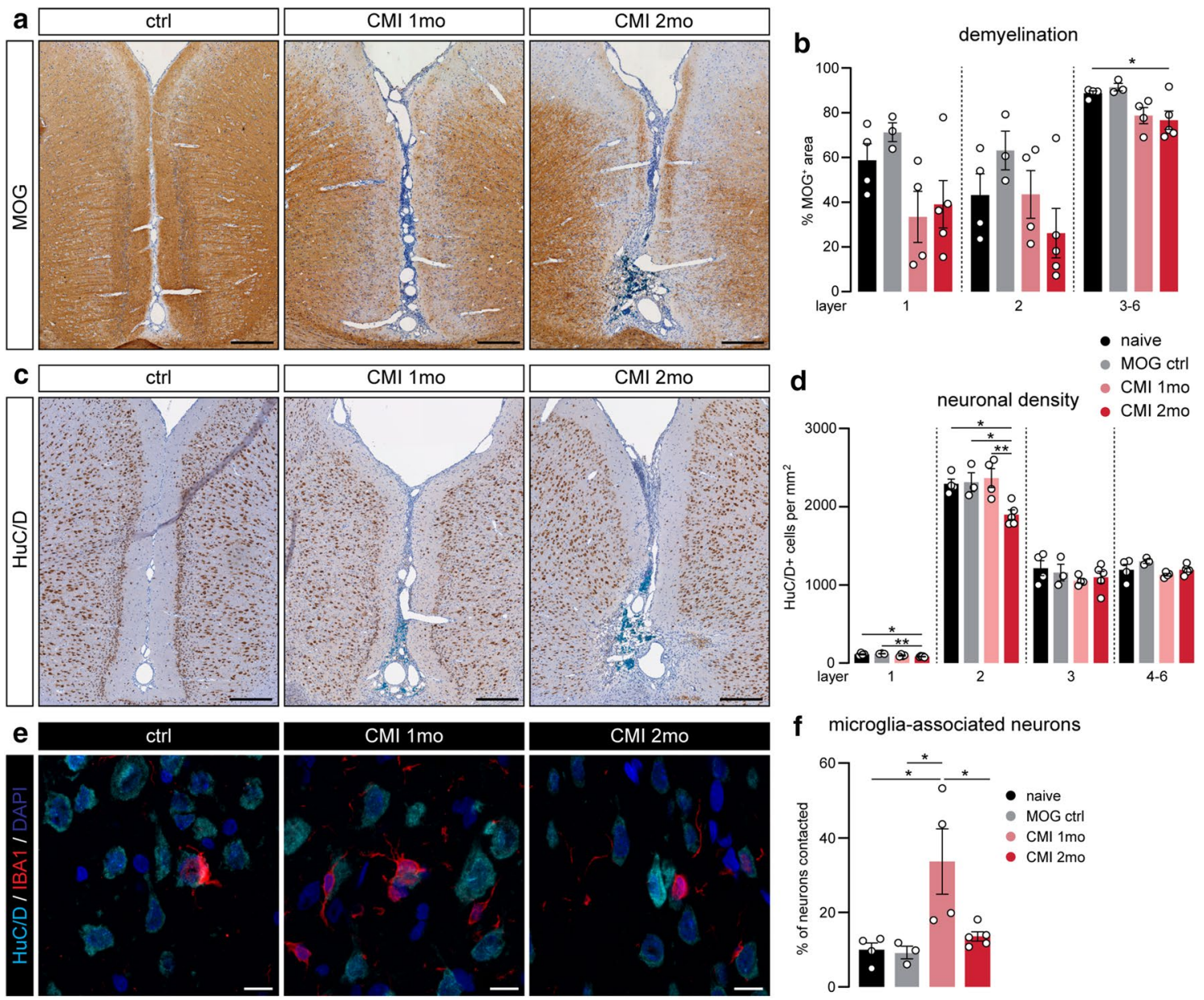

f microglia-associated neurons
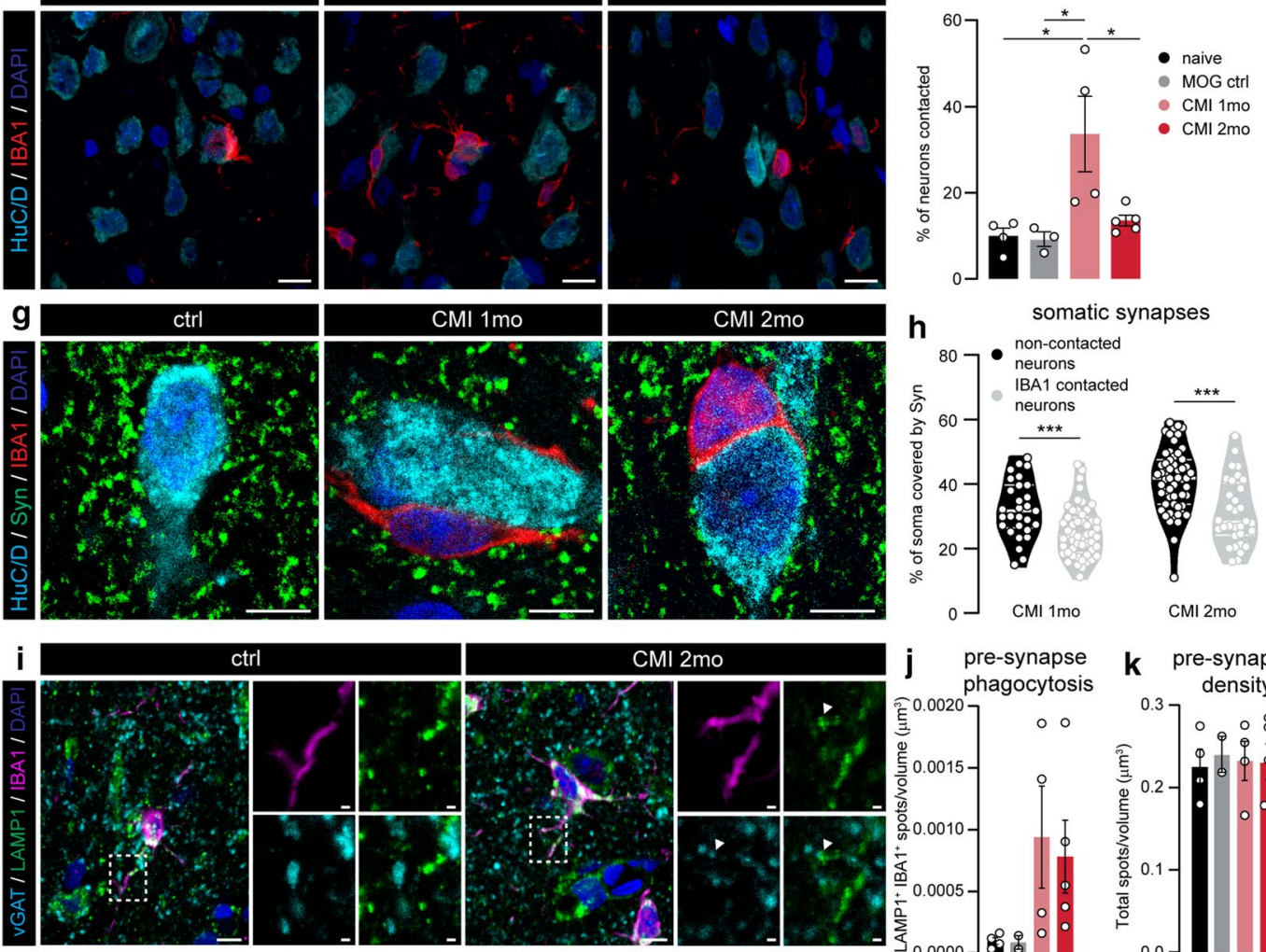

MI $2 \mathrm{mo}$
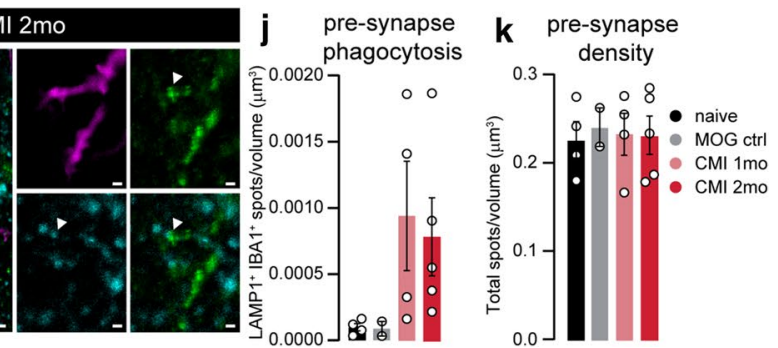

pathological processes in MS meninges and cortex, we are convinced that this model will be a valuable addition to our toolbox for studying progressive MS.

In conclusion, we have uncovered two distinct MSspecific microglial phenotypes in the cortex of progressive MS donors that are driven by local meningeal inflammation and differentially associate with neuronal damage. Results obtained in a novel experimental model for chronic MSlike meningeal inflammation suggest that these phenotypes may occur sequentially and that microglia lose their protective properties over time, leading to neuronal loss. Hence, timely targeting of the processes contributing to microglial 
४Fig. 6 Experimentally induced chronic meningeal inflammation recapitulates cortical pathology in the MS cortex. a Representative images of MOG-stained cortex around the sagittal sulcus of naive, CMI 1 month and CMI 2 months animals. b Quantification of the percentage of $\mathrm{MOG}^{+}$pixels in cortical layers 1,2 and 3-6 surrounding the sagittal sulcus in the four groups of rats. c Representative images showing $\mathrm{HuC} / \mathrm{D}^{+}$neurons in the cortex of naive, CMI 1 month and CMI 2 months animals. d Quantification of neuronal density in the different cortical layers. e Representative maximum projection confocal images of cortical layer 3 from naive, CMI 1 month and CMI 2 months animals double-labelled with IBA1 (microglia) and $\mathrm{HuC} / \mathrm{D}$ (neurons). $\mathbf{f}$ Quantification of the percentage of neuronal somata directly in contact with microglia soma in cortical layer 3. g Representative single z-plane confocal images of cortical layer 3 of naive, CMI 1 month and CMI 2 months animals immunolabelled for HuC/D (neuronal somata), IBA1 (microglia) and Synaptophysin (pre-synapses). $\mathbf{h}$ Quantification of the percentage of neuronal soma covered by Synaptophysin-labelled structures in microgliacontacted neurons and neurons not associated with microglia in CMI animals (pooled data from 3 animals in each group). i Representative images of IBA1 (microglia), LAMP1 (lysosomes) and vGAT (presynapses) expression in cortical layer 3 of a naive and CMI 2 months animal. $\mathbf{j}$ Quantification of the density of $\mathrm{vGAT}^{+}$spots in microglial lysosomes of cortical layer 3 . $\mathbf{k}$ Total density of vGAT spots in layer 3 of the cortex. Individual datapoints indicate averaged data from an individual animal $(\mathbf{b}, \mathbf{d}, \mathbf{f}, \mathbf{h}, \mathbf{j}, \mathbf{k})$, or individual neurons $(\mathbf{h})$, columns and error bars show mean \pm SEM; $* p<0.05$, **p $<0.01$, *** $\mathrm{p}<0.001 ; n=4$ naïve, $n=3 \mathrm{MOG}$ ctrl, $n=4$ CMI 1 month, $n=5 \mathrm{CMI}$ 2 months (b, d, f, h, j, k) with the exception of $n=2$ MOG ctrl in (j, k), $n=80 \mathrm{CMI} 1 \mathrm{mo}$ and $n=88 \mathrm{CMI} 2$ months neurons (h). Scale bars $=250 \mu \mathrm{m}(\mathbf{a}, \mathbf{c}), 10 \mu \mathrm{m}(\mathbf{e}, \mathbf{g})$

activation in the progressive MS cortex provides an interesting therapeutic strategy to combat progressive MS.

Supplementary Information The online version contains supplementary material available at https://doi.org/10.1007/s00401-021-02293-4.

Acknowledgements We thank the Microscopy and Cytometry Core Facility and Mike de Kok of the Amsterdam UMC for excellent technical support. We thank the Mo2Ab Core Facility for providing the HLA class II (OX6) antibody. We thank the UK MS Society Tissue Bank at Imperial College and Dr. Djordje Gveric for the supply of human post-mortem tissue samples. This work was supported by the National MS Society (Grant RG-1601-07456 to RR and JvH) and the Dutch MS Research Foundation MS18-358 to HEdV.

Author contributions $\mathrm{LvO}$ and CRM performed experiments, analyzed data, contributed to design of the study and wrote the manuscript. CPM performed experiments and revised the manuscript. SK, REJ, AK, SMAvdP, LK, IM, ED and MF performed experiments and analyzed data. GJS, JJG, SA and NDM provided valuable scientific input and revised the manuscript. JvH and HEdV contributed to design of the study, obtained research grants and revised the manuscript. RR conceived and supervised the study, obtained research grants and revised the manuscript. MEW conceived and supervised the study, performed experiments, analyzed data and wrote the manuscript.

Open Access This article is licensed under a Creative Commons Attribution 4.0 International License, which permits use, sharing, adaptation, distribution and reproduction in any medium or format, as long as you give appropriate credit to the original author(s) and the source, provide a link to the Creative Commons licence, and indicate if changes were made. The images or other third party material in this article are included in the article's Creative Commons licence, unless indicated otherwise in a credit line to the material. If material is not included in the article's Creative Commons licence and your intended use is not permitted by statutory regulation or exceeds the permitted use, you will need to obtain permission directly from the copyright holder. To view a copy of this licence, visit http://creativecommons. org/licenses/by/4.0/.

\section{References}

1. Arganda-Carreras I, Fernández-González R, Muñoz-Barrutia A, Ortiz-De-Solorzano C (2010) 3D reconstruction of histological sections: Application to mammary gland tissue. Microsc Res Tech 73:1019-1029. https://doi.org/10.1002/jemt.20829

2. Bankhead P, Loughrey MB, Fernández JA, Dombrowski Y, McArt DG, Dunne PD et al (2017) QuPath: Open source software for digital pathology image analysis. Sci Rep. https://doi.org/10.1038/ s41598-017-17204-5

3. Bell L, Lenhart A, Rosenwald A, Monoranu CM, BerberichSiebelt F (2020) Lymphoid aggregates in the CNS of progressive multiple sclerosis patients lack regulatory T cells. Front Immunol 10:1-18. https://doi.org/10.3389/fimmu.2019.03090

4. Butovsky O, Weiner HL (2018) Microglial signatures and their role in health and disease. Nat Rev Neurosci. https://doi.org/10. 1038/s41583-018-0057-5

5. Calabrese M, Magliozzi R, Ciccarelli O, Geurts JJG, Reynolds R, Martin R (2015) Exploring the origins of grey matter damage in multiple sclerosis. Nat Rev Neurosci 16:147-158. https://doi.org/ $10.1038 / \mathrm{nrn} 3900$

6. Calabrese M, Poretto V, Favaretto A, Alessio S, Bernardi V, Romualdi $\mathrm{C}$ et al (2012) Cortical lesion load associates with progression of disability in multiple sclerosis. Brain 135:2952-2961. https://doi.org/10.1093/brain/aws246

7. Chen Z, Jalabi W, Hu W, Park H-J, Gale JT, Kidd GJ et al (2014) Microglial displacement of inhibitory synapses provides neuroprotection in the adult brain. Nat Commun 5:1-12. https://doi.org/ 10.1038/ncomms5486

8. Compston A, Coles A (2008) Multiple sclerosis. Lancet 372:1502-1517

9. Cserép C, Pósfai B, Lénárt N, Fekete R, László ZI, Lele Z et al (2020) Microglia monitor and protect neuronal function through specialized somatic purinergic junctions. Science (80-) 367:528537. https://doi.org/10.1126/science.aax6752

10. Damisah EC, Hill RA, Rai A, Chen F, Rothlin CV, Ghosh S et al (2020) Astrocytes and microglia play orchestrated roles and respect phagocytic territories during neuronal corpse removal in vivo. Sci Adv 6:1-12. https://doi.org/10.1126/sciadv.aba3239

11. Deczkowska A, Keren-Shaul H, Weiner A, Colonna M, Schwartz M, Amit I (2018) Disease-associated microglia: a universal immune sensor of neurodegeneration. Cell 173:1073-1081. https://doi.org/10.1016/j.cell.2018.05.003

12. Dissing-Olesen L, LeDue JM, Rungta RL, Hefendehl JK, Choi HB, MacVicar BA (2014) Activation of neuronal NMDA receptors triggers transient ATP-mediated microglial process outgrowth. J Neurosci 34:10511-10527. https://doi.org/10.1523/ JNEUROSCI.0405-14.2014

13. Di Liberto G, Pantelyushin S, Kreutzfeldt M, Page N, Musardo $\mathrm{S}$, Coras $\mathrm{R}$ et al (2018) Neurons under T cell attack coordinate 
phagocyte-mediated synaptic stripping. Cell. https://doi.org/10. 1016/j.cell.2018.07.049

14. Dutta R, Chang A, Doud MK, Kidd GJ, Ribaudo MV, Young EA et al (2011) Demyelination causes synaptic alterations in hippocampi from multiple sclerosis patients. Ann Neurol 69:445454. https://doi.org/10.1002/ana.22337

15. Eijlers AJC, Van Geest Q, Dekker I, Steenwijk MD, Meijer KA, Hulst HE et al (2018) Predicting cognitive decline in multiple sclerosis: A 5-year follow-up study. Brain 141:2605-2618. https:// doi.org/10.1093/brain/awy202

16. Faissner S, Plemel JR, Gold R, Yong VW (2019) Progressive multiple sclerosis: from pathophysiology to therapeutic strategies. Nat Rev Drug Discov. https://doi.org/10.1038/s41573-019-0035-2

17. Ferreira T, Ou Y, Li S, Giniger E, van Meyel DJ (2014) Dendrite architecture organized by transcriptional control of the F-actin nucleator Spire. Dev 141:650-660. https://doi.org/10.1242/dev. 099655

18. Fontainhas AM, Wang M, Liang KJ, Chen S, Mettu P, Damani $M$ et al (2011) Microglial morphology and dynamic behavior is regulated by ionotropic glutamatergic and GABAergic neurotransmission. PLoS ONE. https://doi.org/10.1371/journal.pone.00159 73

19. Gardner C, Magliozzi R, Durrenberger PF, Howell OW, Rundle J, Reynolds R (2013) Cortical grey matter demyelination can be induced by elevated pro-inflammatory cytokines in the subarachnoid space of MOG-immunized rats. Brain 136:3596-3608. https://doi.org/10.1093/brain/awt279

20. Hauser SL, Oksenberg JR (2006) The neurobiology of multiple sclerosis: genes, inflammation, and neurodegeneration. Neuron 52:61-76

21. Hellwig S, Brioschi S, Dieni S, Frings L, Masuch A, Blank T et al (2016) Altered microglia morphology and higher resilience to stress-induced depression-like behavior in CX3CR1deficient mice. Brain Behav Immun 55:126-137. https://doi.org/ 10.1016/j.bbi.2015.11.008

22. Hinwood M, Tynan RJ, Charnley JL, Beynon SB, Day TA, Walker FR (2013) Chronic stress induced remodeling of the prefrontal cortex: Structural re-organization of microglia and the inhibitory effect of minocycline. Cereb Cortex 23:1784-1797. https://doi.org/10.1093/cercor/bhs 151

23. Howell OW, Reeves CA, Nicholas R, Carassiti D, Radotra B, Gentleman SM et al (2011) Meningeal inflammation is widespread and linked to cortical pathology in multiple sclerosis. Brain 134:2755-2771. https://doi.org/10.1093/brain/awr182

24. James RE, Schalks R, Browne E, Eleftheriadou I, Munoz CP, Mazarakis ND et al (2020) Persistent elevation of intrathecal pro-inflammatory cytokines leads to multiple sclerosis-like cortical demyelination and neurodegeneration. Acta Neuropathol Commun 8:66. https://doi.org/10.1186/s40478-020-00938-1

25. Jürgens T, Jafari M, Kreutzfeldt M, Bahn E, Brück W, Kerschensteiner $\mathrm{M}$ et al (2016) Reconstruction of single cortical projection neurons reveals primary spine loss in multiple sclerosis. Brain 139:39-46. https://doi.org/10.1093/brain/awv353

26. Keren-Shaul H, Spinrad A, Weiner A, Matcovitch-Natan O, Dvir-Szternfeld R, Ulland TK et al (2017) A unique microglia type associated with restricting development of Alzheimer's disease. Cell 169:1276-1290.e17. https://doi.org/10.1016/j.cell. 2017.05.018

27. Krasemann S, Madore C, Cialic R, Ochando J, Haass C, Krasemann $S$ et al (2017) The TREM2-APOE pathway drives the transcriptional phenotype of dysfunctional microglia in neurodegenerative diseases article the TREM2-APOE pathway drives the transcriptional phenotype of dysfunctional microglia in neurodegenerative diseases. Immunity 47:566-581.e9. https://doi. org/10.1016/j.immuni.2017.08.008
28. Lublin FD, Reingold SC, Cohen JA, Cutter GR, Sørensen PS, Thompson AJ et al (2014) Defining the clinical course of multiple sclerosis: The 2013 revisions. Neurology 83:278-286

29. Luchetti S, Fransen NL, van Eden CG, Ramaglia V, Mason M, Huitinga I (2018) Progressive multiple sclerosis patients show substantial lesion activity that correlates with clinical disease severity and sex: a retrospective autopsy cohort analysis. Acta Neuropathol 135:511-528. https://doi.org/10.1007/ s00401-018-1818-y

30. Magliozzi R, Howell O, Vora A, Serafini B, Nicholas R, Puopolo M et al (2007) Meningeal B-cell follicles in secondary progressive multiple sclerosis associate with early onset of disease and severe cortical pathology. Brain 130:1089-1104. https://doi.org/10.1093/brain/awm038

31. Magliozzi R, Howell OW, Reeves C, Roncaroli F, Nicholas R, Serafini B et al (2010) A Gradient of neuronal loss and meningeal inflammation in multiple sclerosis. Ann Neurol 68:477493. https://doi.org/10.1002/ana.22230

32. Magliozzi R, Scalfari A, Pisani AI, Ziccardi S, Marastoni D, Pizzini FB et al (2020) The CSF profile linked to cortical damage predicts multiple sclerosis activity. Ann Neurol 88:562-573. https://doi.org/10.1002/ana.25786

33. Mahad DH, Trapp BD, Lassmann H (2015) Pathological mechanisms in progressive multiple sclerosis. Lancet Neurol 14:183193. https://doi.org/10.1016/S1474-4422(14)70256-X

34. McGeer PL, Itagaki S, Tago H, McGeer EG (1987) Reactive microglia in patients with senile dementia of the Alzheimer type are positive for the histocompatibility glycoprotein HLADR. Neurosci Lett 79:195-200. https://doi.org/10.1016/03043940(87)90696-3

35. Michailidou I, Willems JGP, Kooi E-J, van Eden C, Gold SM, Geurts JJG et al (2015) Complement C1q-C3 associated synaptic changes in multiple sclerosis hippocampus. Ann Neurol. https://doi.org/10.1002/ana.24398

36. Miller DH, Hornabrook RW, Purdie G (1992) The natural history of multiple sclerosis: a regional study with some longitudinal data. J Neurol Neurosurg Psychiatry 55:341-346. https:// doi.org/10.1136/jnnp.55.5.341

37. Morrison H, Young K, Qureshi M, Rowe RK, Lifshitz J (2017) Quantitative microglia analyses reveal diverse morphologic responses in the rat cortex after diffuse brain injury. Sci Rep 7:1-12. https://doi.org/10.1038/s41598-017-13581-z

38. Nimmerjahn A, Kirchhoff F, Helmchen F (2005) Resting microglial cells are highly dynamic surveillants of brain parenchyma in vivo. Science 308:1314-1318. https://doi.org/10.1126/scien ce. 1110647

39. Ohsawa K, Sanagi T, Nakamura Y, Suzuki E, Inoue K, Kohsaka S (2012) Adenosine A3 receptor is involved in ADPinduced microglial process extension and migration. J Neurochem 121:217-227. https://doi.org/10.1111/j.1471-4159.2012. 07693.x

40. Pang Z, Chong J, Li S, Xia J (2020) Metaboanalystr 3.0: toward an optimized workflow for global metabolomics. Metabolites. https://doi.org/10.3390/metabo10050186

41. Peterson JW, Bö L, Mörk S, Chang A, Trapp BD (2001) Transected neurites, apoptotic neurons, and reduced inflammation in cortical multiple sclerosis lesions. Ann Neurol 50:389-400. https://doi.org/10.1002/ana.1123

42. Petrova N, Nutma E, Carassiti D, Newman J, Amor S, Altmann DR et al (2020) Synaptic loss in multiple sclerosis spinal cord. Ann Neurol. https://doi.org/10.1002/ana.25835

43. Picon C, Jayaraman A, James R, Beck C, Gallego P, Witte ME et al (2021) Neuron-specific activation of necroptosis signaling in multiple sclerosis cortical grey matter. Acta Neuropathol. https://doi.org/10.1007/s00401-021-02274-7 
44. Popescu V, Klaver R, Voorn P, Galis-de Graaf Y, Knol D, Twisk $\mathrm{J}$ et al (2015) What drives MRI-measured cortical atrophy in multiple sclerosis? Mult Scler. https://doi.org/10.1177/13524 58514562440

45. Reali C, Magliozzi R, Roncaroli F, Nicholas R, Howell OW, Reynolds R (2020) B cell rich meningeal inflammation associates with increased spinal cord pathology in multiple sclerosis. Brain Pathol. https://doi.org/10.1111/bpa.12841

46. Rüther BJ, Scheld M, Dreymueller D, Clarner T, Kress E, Brandenburg LO et al (2017) Combination of cuprizone and experimental autoimmune encephalomyelitis to study inflammatory brain lesion formation and progression. Glia 65:1900 1913. https://doi.org/10.1002/glia.23202

47. Schirmer L, Velmeshev D, Holmqvist S, Kaufmann M, Werneburg S, Jung D et al (2019) Neuronal vulnerability and multilineage diversity in multiple sclerosis. Nature 573:75-82. https://doi.org/10.1038/s41586-019-1404-Z

48. Serafini B, Rosicarelli B, Magliozzi R, Stigliano E, Aloisi F (2004) Detection of ectopic B-cell follicles with germinal centers in the meninges of patients with secondary progressive multiple sclerosis. Brain Pathol 14:164-174. https://doi.org/10. 1111/j.1750-3639.2004.tb00049.x

49. Sharma K, Wu LJ, Eyo UB (2020) Calming neurons with a microglial touch. Trends Neurosci 43:197-199. https://doi.org/ 10.1016/j.tins.2020.01.008

50. Sierra A, Abiega O, Shahraz A, Neumann H (2013) Janus-faced microglia: beneficial and detrimental consequences of microglial phagocytosis. Front Cell Neurosci 7:6
51. Smith KL, Kassem MS, Clarke DJ, Kuligowski MP, Bedoya-Pérez MA, Todd SM et al (2019) Microglial cell hyper-ramification and neuronal dendritic spine loss in the hippocampus and medial prefrontal cortex in a mouse model of PTSD. Brain Behav Immun 80:889-899. https://doi.org/10.1016/j.bbi.2019.05.042

52. Vogel DYS, Vereyken EJF, Glim JE, Heijnen PDAM, Moeton M, van der Valk P et al (2013) Macrophages in inflammatory multiple sclerosis lesions have an intermediate activation status. J Neuroinflamm. https://doi.org/10.1186/1742-2094-10-35

53. Wegner C, Esiri MM, Chance SA, Palace J, Matthews PM (2006) Neocortical neuronal, synaptic, and glial loss in multiple sclerosis. Neurology 67:960-967. https://doi.org/10.1212/01.wnl.00002 37551.26858 .39

54. Werneburg S, Jung J, Kunjamma RB, Ha S-K, Luciano NJ, Willis $\mathrm{CM}$ et al (2019) Targeted complement inhibition at synapses prevents microglial synaptic engulfment and synapse loss in demyelinating disease. Immunity. https://doi.org/10.1016/j.immuni.2019. 12.004

55. Wu Y, Dissing-Olesen L, MacVicar BA, Stevens B (2015) Microglia: dynamic mediators of synapse development and plasticity. Trends Immunol 36:605-613

Publisher's Note Springer Nature remains neutral with regard to jurisdictional claims in published maps and institutional affiliations.

\section{Authors and Affiliations}

\section{Lynn van Olst ${ }^{1}$. Carla Rodriguez-Mogeda ${ }^{1}$. Carmen Picon ${ }^{2}$. Svenja Kiljan ${ }^{3} \cdot$ Rachel E. James $^{2}$ - Alwin Kamermans ${ }^{1}$. Susanne M. A. van der Pol ${ }^{1} \cdot$ Lydian Knoop $^{4}$ - Iliana Michailidou ${ }^{5}$. Evelien Drost ${ }^{1}$ - Marc Franssen ${ }^{1}$. Geert J. Schenk ${ }^{3}$. Jeroen J. G. Geurts ${ }^{3}$. Sandra Amor ${ }^{4}$. Nicholas D. Mazarakis ${ }^{2}$. Jack van Horssen ${ }^{1} \cdot$ Helga E. de Vries ${ }^{1,6}$. Richard Reynolds ${ }^{2,7} \cdot$ Maarten E. Witte ${ }^{1,4}$}

1 Department of Molecular Cell Biology and Immunology, Amsterdam UMC, MS Center Amsterdam, Amsterdam Neuroscience, Amsterdam, Netherlands

2 Division of Neuroscience, Department of Brain Sciences, Imperial College London, Hammersmith Hospital Campus, Burlington Danes Building, Du Cane Road, London W12 0NN, UK

3 Department of Anatomy and Neurosciences, Amsterdam UMC, MS Center Amsterdam, Amsterdam Neuroscience, Amsterdam, Netherlands

4 Department of Pathology, Amsterdam UMC, MS Center Amsterdam, Amsterdam Neuroscience, Amsterdam, Netherlands
5 Department of Clinical Genetics, Leiden University Medical Center, Leiden, Netherlands

6 Department of Medical Biochemistry, Amsterdam UMC, Amsterdam Cardiovascular Sciences, Amsterdam, The Netherlands

7 Centre for Molecular Neuropathology, LKC School of Medicine, Nanyang Technological University, Singapore, Singapore 